\title{
UWB Localization in a Smart Factory: Augmentation Methods and Experimental Assessment
}

\author{
Luca Barbieri, Student Member, IEEE, Mattia Brambilla, Member, IEEE, Andrea Trabattoni, Stefano Mervic, \\ and Monica Nicoli, Member, IEEE
}

\begin{abstract}
The advent of the fourth industrial revolution (Industry 4.0) aims at increasing automation and efficiency in manufacturing processes by the adoption of information and communication technologies. Several of the proposed solutions rely on precise localization of material, equipment or operators. This article investigates the employment of Ultra WideBand (UWB) real-time location systems (RTLS) in a factory environment and proposes an augmentation technique to mitigate the impairments that arise in such a complex scenario. A Bayesian filtering method is developed to jointly track the motion dynamics and the time-varying visibility conditions of the UWB antennas, with particle-based implementation to deal with the non-linearity of the UWB measurements. Laboratory tests and industrial experiments are carried out to evaluate the performance of three commercial off-the-shelf UWB technologies, namely Decawave, Sewio and Ubisense. The experimental data are then used to calibrate and test the developed filtering technique, showing that it is possible to significantly reduce the positioning error originating from dense multipath and NLOS effects by jointly tracking the target dynamics and visibility conditions.
\end{abstract}

Index Terms-UWB, Industrial Localization, NLOS Compensation, Bayesian Tracking, Jump Markov System, Particle Filter

\section{INTRODUCTION}

$\mathbf{F}$ OR decades, Ultra WideBand (UWB) radar has been used in military and civilian applications for high-resolution sensing and imaging [1]. Nowadays, this technology is being considered as a viable solution for precise localization, especially for Global Navigation Satellite System (GNSS)denied environments. Compared to other widespread localization technologies, such as Bluetooth, Zigbee and RFID, UWB has the ability to provide enhanced ranging accuracy [2]-[5], multipath resolution and robustness to interference [1], [6], [7] thanks to the large bandwidth. These technical characteristics make the UWB technology suitable for a variety of emerging location-based services, ranging from consumer to Industrial Internet of Things (IIoT) applications. Regarding the former, UWB modules have been released for smartphones since 2019 to provide enhanced spatial awareness services [8], enabling a potential massive market penetration. In IIoT context, likewise, accurate location information jointly with gigabit communication capability are interesting features for autonomous industrial systems. This led many companies, such as Decawave, Sewio, OpenRTLS, Time Domain, Ubisense and Zebra, to

This work was supported by the project Total Efficiency 4.0 funded by the Regione Lombardia grant POR-FESR 2014-2020 ID 235307. develop proprietary commercial UWB solutions operating over unlicensed bands.

Though the application is extremely relevant and timely due to the emergence of Industry 4.0, as of today very few experimental studies have been performed to assess the performances of UWB-based Real-Time Location Systems (RTLS) in real industrial environments [9]-[20]. In [9], a first comparative analysis is carried out with Decawave, Bespoon and OpenRTLS. A similar study is presented in [10], where Decawave devices are analyzed in various environments. Decawave suite is compared to Bespoon in [11] and to Ubisense in [12] for various propagation conditions, using a Particle Filter (PF) technique, fitted to the Time of Arrival (ToA) experimental data. Decawave DW1000 modules are analyzed also in [13], where the ranging accuracy is evaluated in an heavy machines laboratory, while the MDEK1000 is evaluated in [14], [15]. Several asynchronous UWB localization solutions are presented in [16]-[18], while [19] and [20] focus on anchor calibration and error correction in UWB networks, respectively. Lastly, a joint Radio Frequency Identification (RFID) and UWB system is developed in [21] to deliver enhanced location awareness in intelligent warehouse management systems.

A main critical issue emerging from all the above studies is the severe degradation due to multipath and Non Line of Sight (NLOS) propagation in real industrial facilities, typically filled with metallic obstacles (e.g., robots, machinery and cabinets) [22], [23]. To mitigate the effects, a decision theoretic framework has been proposed in [24] to discriminate Line of Sight (LOS)/NLOS conditions using perfect or partial knowledge of ToA distribution. A statistical characterization of the Channel Impulse Response (CIR) for NLOS identification is employed in [25], [26], while other mitigation approaches rely on Bayesian filtering embedding statistical information on the propagation conditions [27], [28], biased or mixture models [29], [30], map information [31], [32], sensor fusion [33]-[35] or variational techniques with skewed/heavy-tail distributions [36]-[40]. Machine learning techniques have been recently emerging as candidate solutions, where set of features are chosen so as to capture NLOS characteristics [41], [42]. A brand new research trend is also $5 \mathrm{G}$ positioning at millimeter waves (mmWave), where ultra-wide spectrum portions [43], [44] can be exploited to enhance the time resolution and enable Simultaneous Localization and Mapping (SLAM) [45], [46]. Furthermore, the use of massive Multiple-Input Multiple- 
Output (MIMO) leads to precise Angle of Arrival (AoA) measurements, facilitating the resolution of NLOS components in the spatial domain, thus improving the localization accuracy [47], [48].

The main limit of the above discussed techniques is the need of either low-level (raw) data, such as CIR, which might not be accessible in commercial off-the-shelf UWB solutions, or of specialized hardware, such as massive MIMO. It is goal of this work to provide an augmentation approach that embeds the information coming from the environment, rather than relying on the raw signals or ad-hoc technologies. This makes the proposed technique suitable for a wide range of legacy UWB systems. The proposed approach mitigates the multipath impairments by a Bayesian filtering technique that operates on the pseudo-ranges affected by NLOS bias and gathered by the UWB devices. Similarly to [28], the method uses a Markovian switching model to handle the LOS/NLOS transitions. With respect to [28], however, here the tracking filter is redesigned to work with Time Difference of Arrival (TDoA) rather than baseband Power Delay Profile (PDP) signals, making the proposed solution suited for integration with commercial UWB systems. Moreover, it is more computationally efficient and scalable for increasing number of APs/tags, as it requires to handle only few scalar TDoA quantities rather than the full PDP for each UWB link. The proposed technique relies on a Jump Markov System (JMS) to jointly track the tag motion dynamics and the LOS/NLOS sight conditions experienced over the UWB links. The undesired, yet unavoidable, range offset due to NLOS propagation is modeled by an exponential random process, which accounts for the UWB signal reflections from the environment. The proposed approach assumes that the NLOS excess delay is a stationary random process, i.e., with statistics that are spatially invariant over the whole environment. This is an approximation, as the NLOS bias might be non stationary but only a high-complexity approach could catch the location-specific multipath configuration (e.g., a ray tracer). Other methods, such as [31], [32], can be employed to compute the NLOS bias all over the space, however, they are typically computationally expensive since a dense point grid is required for building the error map. On the other hand, the proposed method is shown to be robust and have affordable complexity, and it requires only the calibration of few parameters, namely the ones characterizing the NLOS distribution. Another feature of the proposed model is that it can be generalized to incorporate other distributions that might characterize different environments, resulting in a resilient and adaptive tracking filter to be considered for enhancing localization accuracy in complex industrial environments.

As a second contribution, we present a set of experimental tests conducted with commercial UWB devices in both laboratory and industrial environments, namely Decawave [49], Ubisense [50] and Sewio [51]. Compared to our preliminary work [52], here we propose the JMS augmentation technique, we extend the experimental study with additional tests and we validate the proposed approach on the overall collected data. The availability of experimental data allows us to calibrate the stochastic model of the proposed approach in order to better adhere the IIoT context, as well as to validate the

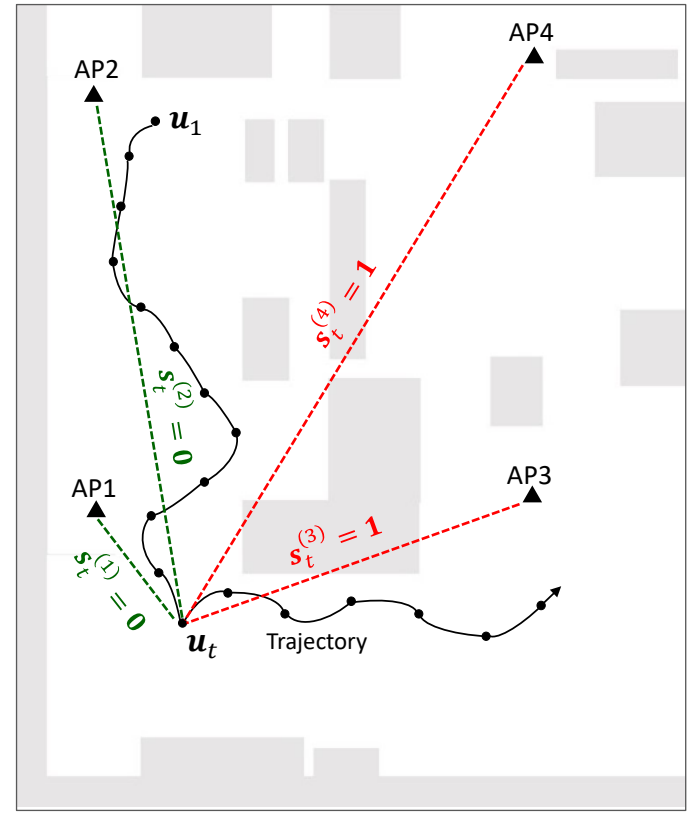

Fig. 1. UWB localization in a real factory (Pirelli Tyre, Milan): 4 APs localize a moving tag in an indoor environment with industrial machines and materials (gray areas). LOS links are in green, while the NLOS ones in red. Dimension of the area: $16 \times 14 \mathrm{~m}$.

algorithm in real industrial conditions. Experimental results show significant performance gains compared to state-of-theart methods for NLOS compensation [36], [37] in factory environments with high density of metallic scatterers and dynamic conditions due to ongoing production activities.

The paper is organized as follows. The proposed augmentation solution is described in Sec. II. Sec. III provides the technical characteristics of the selected UWB devices, while the experimental tests are illustrated in Sec. IV. Sec. V provides the validation of the proposed tracking technique on collected raw data and Sec. VI draws conclusions.

Notation: bold letters indicate column vectors which can belong to the set of real numbers $\mathbb{R}$ or the set of naturals $\mathbb{N}$. Symbol $\mathbf{I}_{N}$ identifies an identity matrix of size $N \times N$, while $\mathbf{0}_{N}$ stands for a null vector of size $N \times 1$. Matrix transposition operator is $(\cdot)^{T}$. Symbol $|\cdot|$ denotes cardinality of a set, while $\|\cdot\|$ is the Euclidean norm. Probability is indicated with $P(\cdot)$. Throughout the paper, the probability density function (pdf) of a random variable $x$, with mean $\mu$, can be shaped according to different well-known distributions. We indicate with $\mathcal{N}\left(x ; \mu, \sigma^{2}\right)$ a Gaussian pdf with variance $\sigma^{2}$, with $\mathcal{L}(x ; \mu, b)$ a Laplace pdf with diversity parameter $b$, with $\mathcal{E}(x ; \lambda)$ an exponential pdf with rate $\lambda$, and with $S T\left(x ; \mu, \sigma^{2}, \delta, \nu\right)$ a Skew $\mathrm{t}$ distribution [36, eq. (1)] with spread $\sigma$, shape parameter $\delta$ and $\nu$ degrees of freedom.

\section{Augmentation System FOR NLOS Mitigation}

In this section, we present the localization model (Sec. II-A) and the Bayesian framework used for deriving the tracking filter (Sec. II-B), followed by an illustrative example revealing the intuition of the proposed approach (Sec. II-C). 


\section{A. Localization Model}

We consider a $2 \mathrm{D}$ localization scenario as illustrated in Fig. 1, where a tag moves in an UWB infrastructured area $\mathcal{U} \subset \mathbb{R}^{2}$ and periodically sends pilot UWB signals to $L \geq 3$ Access Points (AP)s located at known fixed positions, which are in charge of estimating tag's position by multi-lateration and/or multi-angulation. The static position of the $\ell$-th AP, with $\ell=1, \ldots, L$, is $\mathbf{p}_{\ell}=\left[p_{\ell, x} p_{\ell, y}\right]^{T} \in \mathcal{U}$, while the mobile tag is indicated by the time-variant (over time $t$ ) position vector $\mathbf{u}_{t}=\left[u_{x, t} u_{y, t}\right]^{T} \in \mathcal{U}$. The dynamics of $\mathbf{u}_{t}$ are modeled as a first-order Markov process as:

$$
\mathbf{u}_{t}=f_{V}\left(\mathbf{u}_{t-1}\right)+\mathbf{w}_{t}=\mathbf{u}_{t-1}+\mathbf{v}_{t} \cdot \Delta t+\mathbf{w}_{t},
$$

where $\mathbf{v}_{t}=\left[v_{x, t} v_{y, t}\right]^{T}$ is the $2 \mathrm{D}$ velocity, $\Delta t$ the sampling interval and $\mathbf{w}_{t}$ the driving noise with known pdf $f_{W}\left(\mathbf{w}_{t}\right)$. We consider a zero-mean Gaussian driving process with standard deviation $\sigma_{w}$ (to be calibrated according to the specific target dynamics) on both $x$ and $y$ axes, which leads to $f_{W}\left(\mathbf{w}_{t}\right)=\mathcal{N}\left(\mathbf{w}_{t} ; \mathbf{0}_{2}, \mathbf{C}_{u}\right)$, with $\mathbf{C}_{u}=\sigma_{w}^{2} \mathbf{I}_{2}$. The tag transition pdf between two consecutive time instants is $p\left(\mathbf{u}_{t} \mid \mathbf{u}_{t-1}\right)=f_{W}\left(\mathbf{u}_{t}-\mathbf{u}_{t-1}-\mathbf{v}_{t} \cdot \Delta t\right)$, and it has to be defined and calibrated for each specific application. Here we assume to know the $2 \mathrm{D}$ mean velocity vector $\mathbf{v}_{t}$, as it can be extracted from embedded Inertial Measurement Unit (IMU) sensors available in commercial UWB systems. Nevertheless the proposed methodology is general enough to be applied to any motion dynamics of the target.

Following the approach in [28], we propose to estimate and compensate NLOS related biases by adding a sight variable into the Bayesian state and leveraging this additional information to counterbalance APs heavily affected by propagation impairments. The sight condition between the tag and the $\ell$-th AP is represented by a first-order Markov chain $s_{t}^{(\ell)}$ with value $s_{t}^{(\ell)}=0$ for LOS and $s_{t}^{(\ell)}=1$ for NLOS and transition probabilities: $p_{0}=P\left(s_{t}^{(\ell)}=0 \mid s_{t-1}^{(\ell)}=0\right)=P\left(s_{t}^{(\ell)}=1 \mid s_{t-1}^{(\ell)}=1\right)$ and $p_{1}=P\left(s_{t}^{(\ell)}=1 \mid s_{t-1}^{(\ell)}=0\right)=P\left(s_{t}^{(\ell)}=0 \mid s_{t-1}^{(\ell)}=1\right)$. The values of $p_{0}$ and $p_{1}$ are environment-specific and have to be calibrated for the specific application.

This model assumes that the sight condition $s_{t}^{(\ell)}$ evolution over time is independent of the tag position $\mathbf{u}_{t}$ as well as of another sight condition $s_{t}^{(k)}$ of $\mathrm{AP} k=1, \ldots, L$, with $k \neq \ell$. Despite the sight condition $s_{t}^{(\ell)}$ is related to $\mathbf{u}_{t}$, this models allows to statistically describe its evolution in a simple, yet efficient, way. It follows that there are no constraints on the type of the tag trajectory as $\mathbf{u}_{t}$ may assume any value. Assuming mutual independence of APs sight conditions, the overall sight process is a first-order Markov chain with transition probabilities:

$$
P\left(\mathbf{s}_{t}=\mathbf{k} \mid \mathbf{s}_{t-1}=\mathbf{h}\right)=\prod_{\ell=1}^{L} P\left(s_{t}^{(\ell)}=k_{\ell} \mid s_{t-1}^{(\ell)}=h_{\ell}\right),
$$

for $\mathbf{k}=\left[k_{1} \cdots k_{L}\right]^{T}$ and $\mathbf{h}=\left[h_{1} \cdots h_{L}\right]^{T}$, with $\mathbf{k}, \mathbf{h} \in \mathcal{S}=$ $\{0,1\}^{L}$.

At time $t$, the estimated range between the tag and AP $\ell$ is modeled as:

$$
\rho_{t}^{(\ell)}=d_{t}^{(\ell)}+s_{t}^{(\ell)} \Delta d_{t}^{(\ell)}+n_{t}^{(\ell)},
$$

where $d_{t}^{(\ell)}=\left\|\mathbf{u}_{t}-\mathbf{p}_{\ell}\right\|$ is the true distance, $n_{t}^{(\ell)}$ is the measurement noise and $\Delta d_{t}^{(\ell)}$ is the excess range due to NLOS. The noise term $n_{t}^{(\ell)}$ is modeled as a Gaussian random variable with mean and variance that depend on the specific sight condition [53] as follows. For LOS (i.e., $s_{t}^{(\ell)}=0$ ), it is modeled as $p\left(n_{t}^{(\ell)}\right)=\mathcal{N}\left(n_{t}^{(\ell)} ; 0, \sigma_{\text {LOS }}^{2}\right)$ while in case of NLOS (i.e., $\left.s_{t}^{(\ell)}=1\right) p\left(n_{t}^{(\ell)}\right)=\mathcal{N}\left(n_{t}^{(\ell)} ; \mu_{n}, \sigma_{\text {NLOS }}^{2}\right)$, where $\mu_{n}$ is the mean excess range. On the other hand, the NLOS excess range $p\left(\Delta d_{t}^{(\ell)}\right)=\mathcal{E}\left(\Delta d_{t}^{(\ell)} ; 1 / \sigma_{d}\right)$ is modeled as an exponential random variable with rate $1 / \sigma_{d}$, in line with the widespread literature on UWB channel modeling in indoor environments [54]-[57]. This model is assessed over the considered environment, allowing us to compensate the NLOS effects in any position of the space. In our analysis, we assume that the noise $n_{t}^{(\ell)}$ is independent of the distance $d_{t}^{(\ell)}$, of the range estimated by any other AP $k \neq \ell$ and of the excess range $\Delta d_{t}^{(\ell)}$. Furthermore, the range errors over different links have the same statistics, i.e., $\left\{\sigma_{\mathrm{LOS}}^{2}, \sigma_{\mathrm{NLOS}}^{2}, \mu_{n}, \sigma_{d}\right\}$ do not vary with AP index $\ell$.

The TDoA measurement $\rho_{t}^{(\ell, k)}=\rho_{t}^{(\ell)}-\rho_{t}^{(k)}$ is computed as difference between ToAs of links $\ell$ and $k$, with $\ell \neq k$ and its modeling accounts for any combination of sight conditions $s_{t}^{(\ell)}$ and $s_{t}^{(k)}$. The possible values it can assume are indicated in Table I, where $d_{t}^{(\ell, k)}=d_{t}^{(\ell)}-d_{t}^{(k)}, n_{t}^{(\ell, k)}=n_{t}^{(\ell)}-n_{t}^{(k)}$, $\Delta d_{t}^{(\ell, k)}=\Delta d_{t}^{(\ell)}-\Delta d_{t}^{(k)}, \sigma_{1}^{2}=2 \sigma_{\mathrm{LOS}}^{2}, \sigma_{2}^{2}=\sigma_{\mathrm{LOS}}^{2}+\sigma_{\mathrm{NLOS}}^{2}$ and $\sigma_{3}^{2}=2 \sigma_{\mathrm{NLOS}}^{2}$. For $s_{t}^{(k)}=s_{t}^{(\ell)}=1, \Delta d_{t}^{(\ell, k)}$ is computed as the difference between two i.i.d. exponential distributions, i.e., it is a Laplace random variable $\mathcal{L}\left(\Delta d_{t}^{(\ell, k)} ; 0, b\right)$ with diversity parameter $b=\sigma_{d}$ [58]. We recall that a random variable $x$ is Laplace distributed with mean $m$ and diversity parameter $b$ if its pdf is described as:

$$
\mathcal{L}(x ; m, b)=\frac{1}{2 b} \exp \left(-\frac{|x-m|}{b}\right) .
$$

Besides TDoA, we also consider AoA measurements for UWB positioning. In a $3 \mathrm{D}$ space, the angular information includes the azimuth AoA $\alpha_{t}^{(\ell)}$ and the elevation AoA $\beta_{t}^{(\ell)}$, which are defined as:

$$
\begin{aligned}
\alpha_{t}^{(\ell)} & =\arctan \left(\left(p_{\ell, y}-u_{y, t}\right) /\left(p_{\ell, x}-u_{x, t}\right)\right), \\
\beta_{t}^{(\ell)} & =\arcsin \left(\left(p_{\ell, z}-u_{z, t}\right) / d_{t}^{(\ell)}\right) .
\end{aligned}
$$

We denote with $\rho_{\alpha, t}^{(\ell)}=\alpha_{t}^{(\ell)}+n_{\alpha, t}^{(\ell)}$ and $\rho_{\beta, t}^{(\ell)}=\beta_{t}^{(\ell)}+n_{\beta, t}^{(\ell)}$, the available measurements of azimuth and elevation AoA, respectively, where $n_{\alpha, t}^{(\ell)}$ and $n_{\beta, t}^{(\ell)}$ are the measurement noises. As in [56], [59]-[61], we consider the AoA measurement error as a Laplace random variables, i.e., $n_{\alpha, t}^{(\ell)} \sim \mathcal{L}\left(\alpha_{t}^{(\ell)}, 0, b_{\alpha}\right)$ and $n_{\beta, t}^{(\ell)} \sim \mathcal{L}\left(\beta_{t}^{(\ell)}, 0, b_{\beta}\right)$.

Considering without loss of generality the AP $\ell=1$ as reference, the set of TDoA measurements available at time $t$ is $\boldsymbol{\rho}_{t}=\left[\rho_{t}^{(1,2)} \cdots \rho_{t}^{(1, L)}\right]^{T}$, which depends on the sight conditions $\mathbf{s}_{t}=\left[s_{t}^{(1)} \cdots s_{t}^{(L)}\right]^{T}$ of all APs. The set of AoA measurements available at time $t$ is $\boldsymbol{\rho}_{\alpha, t}=\left[\rho_{\alpha, t}^{(1)} \cdots \rho_{\alpha, t}^{(L)}\right]^{T}$, which does not depend on the sight condition values. The overall set of measurements available at time $t$ is thus $\boldsymbol{\Omega}_{t}=$ $\left[\boldsymbol{\rho}_{t}^{T} \boldsymbol{\rho}_{\alpha, t}^{T}\right]^{T}$, where $\boldsymbol{\rho}_{t}$ and $\boldsymbol{\rho}_{\alpha, t}$ are independent. 
TABLE I

MODELING OF THE TDOA MEASUREMENT $\rho_{t}^{(\ell, k)}$ WITH RESPECT TO THE DIFFERENT PROPAGATION CONDITIONS OF APS $\ell$ AND $k$

\begin{tabular}{lllll}
$\rho_{t}^{(\ell, k)}$ & $s_{t}^{(\ell)} s_{t}^{(k)} p\left(n_{t}^{(\ell, k)}\right)$ & $p\left(\Delta d_{t}^{(\ell, k)}\right)$ \\
\hline$d_{t}^{(\ell, k)}+n_{t}^{(\ell, k)}$ & 0 & 0 & $\mathcal{N}\left(n_{t}^{(\ell, k)} ; 0, \sigma_{1}^{2}\right)$ & - \\
$d_{t}^{(\ell, k)}+\Delta d_{t}^{(\ell)}+n_{t}^{(\ell, k)}$ & 1 & 0 & $\mathcal{N}\left(n_{t}^{(\ell, k)} ; \mu_{n}, \sigma_{2}^{2}\right)$ & $\mathcal{E}\left(\Delta d_{t}^{(\ell)} ; 1 / \sigma_{d}\right)$ \\
$d_{t}^{(\ell, k)}-\Delta d_{t}^{(k)}+n_{t}^{(\ell, k)}$ & 0 & 1 & $\mathcal{N}\left(n_{t}^{(\ell, k)} ;-\mu_{n}, \sigma_{2}^{2}\right) \mathcal{E}\left(-\Delta d_{t}^{(k)} ; 1 / \sigma_{d}\right)$ \\
$d_{t}^{(\ell, k)}+\Delta d_{t}^{(\ell, k)}+n_{t}^{(\ell, k)} 1$ & 1 & $\mathcal{N}\left(n_{t}^{(\ell, k)} ; 0, \sigma_{3}^{2}\right)$ & $\mathcal{L}\left(\Delta d_{t}^{(\ell, k)} ; 0, b\right)$ \\
\hline
\end{tabular}

As a whole, the localization process is described as a combination of a JMS and an Hidden Markov Model (HMM). The state $\mathbf{x}_{t}$ is hidden into the TDoA measurements $\boldsymbol{\rho}_{t}$ with dynamics controlled by the sight conditions $\mathbf{s}_{t}$. The state $\mathbf{x}_{t}$ is also hidden into the AoA measurements $\boldsymbol{\rho}_{\alpha, t}$ but the dynamics are not controlled by the sight conditions since the LOS/NLOS contribution is modeled by a unique Laplace distribution. To estimate $\mathbf{u}_{t}$, it is necessary to also track the variations of $\mathbf{s}_{t}$, thereby we propose a joint Bayesian estimation of the positionsight state $\mathbf{x}_{t}=\left(\mathbf{u}_{t}, \mathbf{s}_{t}\right)$, as described in the next section.

\section{B. Bayesian Tracking Method of the Joint Position-Sight State}

The estimate of $\mathbf{x}_{t}$ based on the observations up to time $t$, i.e., $\boldsymbol{\Omega}_{1: t}=\left[\boldsymbol{\Omega}_{1}^{T} \cdots \boldsymbol{\Omega}_{t}^{T}\right]^{T}$, is obtained following a Bayesian approach. This leads to the evaluation of the a-posteriori pdf $p\left(\mathbf{x}_{t} \mid \boldsymbol{\Omega}_{1: t}\right)$ as a combination of the a-priori pdf $p\left(\mathbf{x}_{t} \mid \boldsymbol{\Omega}_{1: t-1}\right)$ and the conditioned pdf $p\left(\boldsymbol{\Omega}_{t} \mid \mathbf{x}_{t}\right)$ as:

$$
p\left(\mathbf{x}_{t} \mid \boldsymbol{\Omega}_{1: t}\right) \propto p\left(\boldsymbol{\Omega}_{t} \mid \mathbf{x}_{t}\right) p\left(\mathbf{x}_{t} \mid \boldsymbol{\Omega}_{1: t-1}\right) .
$$

The a-priori pdf is evaluated for $t>1$ according to the Chapman-Kolmogorov equation [62] incorporating the tag motion model (1) and the Markov chain for sight condition as:

$$
\begin{aligned}
& p\left(\mathbf{x}_{t} \mid \boldsymbol{\Omega}_{1: t-1}\right)= \\
& \quad \sum_{\mathbf{s}_{t-1} \in \mathcal{S}} P\left(\mathbf{s}_{t} \mid \mathbf{s}_{t-1}\right) \int_{\mathcal{U}} p\left(\mathbf{u}_{t} \mid \mathbf{u}_{t-1}\right) p\left(\mathbf{x}_{t-1} \mid \boldsymbol{\Omega}_{1: t-1}\right) \mathrm{d} \mathbf{u}_{t-1},
\end{aligned}
$$

while for $t=1, p\left(\mathbf{x}_{1} \mid \boldsymbol{\Omega}_{0}\right)=p\left(\mathbf{u}_{0}\right) P\left(\mathbf{s}_{0}\right)$ accounts for the available a priori information about the initial sight conditions and position. To ease the notation, the action sign has been omitted from (8).

To compute the conditioned pdf, it is important to recall that the $L-1$ TDoA and $L$ AoA observations are conditionally independent. This follows from the independence assumption among the sight conditions, as well as between the TDoA and AoA observations. Furthermore, the state $\mathbf{x}_{t}$ is not directly observable from the observations. Following these considerations, the conditioned pdf is computed as:

$$
p\left(\boldsymbol{\Omega}_{t} \mid \mathbf{x}_{t}\right)=\prod_{k=2}^{L} p\left(\rho_{t}^{(1, k)} \mid \mathbf{u}_{t}, s_{t}^{(1)}, s_{t}^{(k)}\right) \prod_{\ell=1}^{L} p\left(\rho_{\alpha, t}^{(\ell)} \mid \mathbf{u}_{t}\right)
$$

where each likelihood $p\left(\rho_{t}^{(1, k)} \mid \mathbf{u}_{t}, s_{t}^{(1)}, s_{t}^{(k)}\right)$ is derived from the measurement model in Table I. Due to this modeling, each combination of LOS/NLOS condition influence the corresponding pdf as:

$$
\begin{aligned}
& p\left(\rho_{t}^{(1, k)} \mid \mathbf{u}_{t}, s_{t}^{(1)}=0, s_{t}^{(k)}=0\right)=\mathcal{N}\left(\rho_{t}^{(1, k)} ; d_{t}^{(1, k)}, \sigma_{1}^{2}\right),(10) \\
& p\left(\rho_{t}^{(1, k)} \mid \mathbf{u}_{t}, s_{t}^{(1)}=1, s_{t}^{(k)}=0\right)= \\
& \int \mathcal{N}\left(\rho_{t}^{(1, k)} ; d_{t}^{(1, k)}+\Delta d_{t}^{(1)}+\mu_{n}, \sigma_{2}^{2}\right) \mathcal{E}\left(\Delta d_{t}^{(1)} ; 1 / \sigma_{d}\right) \mathrm{d} \Delta d_{t}^{(1)}, \\
& p\left(\rho_{t}^{(1, k)} \mid \mathbf{u}_{t}, s_{t}^{(1)}=0, s_{t}^{(k)}=1\right)= \\
& \int \mathcal{N}\left(\rho_{t}^{(1, k)} ; d_{t}^{(1, k)}-\Delta d_{t}^{(k)}-\mu_{n}, \sigma_{2}^{2}\right) \mathcal{E}\left(-\Delta d_{t}^{(k)} ; 1 / \sigma_{d}\right) \mathrm{d} \Delta d_{t}^{(k)}, \\
& p\left(\rho_{t}^{(1, k)} \mid \mathbf{u}_{t}, s_{t}^{(1)}=1, s_{t}^{(k)}=1\right)= \\
& \int \mathcal{N}\left(\rho_{t}^{(1, k)} ; d_{t}^{(1, k)}+\Delta d_{t}^{(1, k)}, \sigma_{3}^{2}\right) \mathcal{L}\left(\Delta d_{t}^{(1, k)} ; 0, \sigma_{d}\right) \mathrm{d} \Delta d_{t}^{(1, k)} .
\end{aligned}
$$

The integrals in (11) and (12) can be computed in closed form using the Exponentially Modified Gaussian (EMG) distribution [63]. More specifically, equation (11) reduces to:

$$
\begin{aligned}
& p\left(\rho_{t}^{(1, k)} \mid \mathbf{u}_{t}, s_{t}^{(1)}=1, s_{t}^{(k)}=0\right)= \\
& \frac{1}{2 \sigma_{d}} e^{\frac{1}{2 \sigma_{d}}\left(2 \mu_{n}+\frac{\sigma_{2}^{2}}{\sigma_{d}}-2 \rho_{t}^{(1, k)}\right)} \operatorname{erfc}\left(\frac{\mu_{n}+\frac{\sigma_{2}^{2}}{\sigma_{d}}-2 \rho_{t}^{(1, k)}}{\sqrt{2} \sigma_{2}}\right),
\end{aligned}
$$

where erfc is the complementary error function, while for (12) we have:

$$
\begin{aligned}
& p\left(\rho_{t}^{(1, k)} \mid \mathbf{u}_{t}, s_{t}^{(1)}=0, s_{t}^{(k)}=1\right)= \\
& \frac{1}{2 \sigma_{d}} e^{\frac{1}{2 \sigma_{d}}\left(-2 \mu_{n}+\frac{\sigma_{2}^{2}}{\sigma_{d}}+2 \rho_{t}^{(1, k)}\right)} \operatorname{erfc}\left(\frac{-\mu_{n}+\frac{\sigma_{2}^{2}}{\sigma_{d}}+2 \rho_{t}^{(1, k)}}{\sqrt{2} \sigma_{2}}\right) .
\end{aligned}
$$

On the other hand, the integral in (13) can be evaluated using the Laplace-Normal (LN) pdf, arising as the sum between independent Laplace and Gaussian random variables as [64]:

$$
\begin{aligned}
& p\left(\rho_{t}^{(1, k)} \mid \mathbf{u}_{t}, s_{t}^{(0)}=1, s_{t}^{(k)}=1\right)= \\
& \frac{1}{2 \sigma_{d}} \Phi\left(\frac{\rho_{t}^{(1, k)}}{\sigma_{3}}\right)\left[\mathrm{R}\left(\frac{\sigma_{3}}{\sigma_{d}}-\frac{\rho_{t}^{(1, k)}}{\sigma_{3}}\right)+\mathrm{R}\left(\frac{\sigma_{3}}{\sigma_{d}}+\frac{\rho_{t}^{(1, k)}}{\sigma_{3}}\right)\right],
\end{aligned}
$$

where $\Phi(\cdot)$ is a zero-mean Gaussian pdf with unitary variance and $\mathrm{R}(x)$ is the Mill's ratio defined as:

$$
\mathrm{R}(x)=\frac{1-\frac{1}{2}\left[1+\operatorname{erf}\left(\frac{x}{\sqrt{2}}\right)\right]}{\Phi(x)},
$$

while $\operatorname{erf}(x)=1+\operatorname{erfc}(x)$ is the error function.

Regarding the azimuth AoA, the likelihood $p\left(\alpha_{t}^{(\ell)} \mid \mathbf{u}_{t}\right)$ is computed as:

$$
p\left(\rho_{\alpha, t}^{(\ell)} \mid \mathbf{u}_{t}\right)=\mathcal{L}\left(\rho_{\alpha, t}^{(\ell)} ; \alpha_{t}^{(\ell)}, b_{\alpha}\right),
$$

i.e., as a Laplace pdf centered around the true azimuth.

The a-posteriori distribution of $\mathbf{x}_{t}$ is evaluated for any $t$ through the recursive computation of (7)-(8). The estimate 


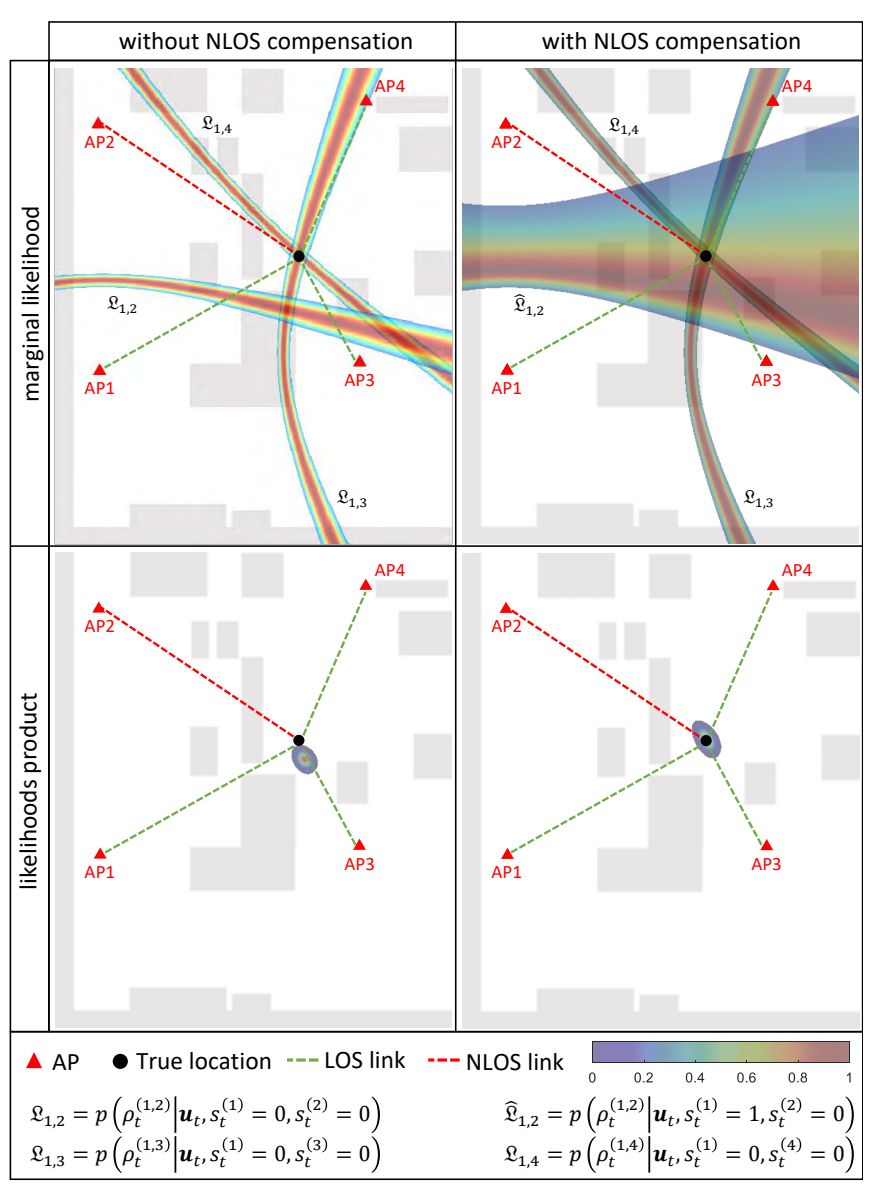

Fig. 2. Comparison of likelihoods for tracking filter with (right) and without NLOS compensation (left). The analysis shows the marginal likelihood functions (top) and the product (bottom).

$\hat{\mathbf{x}}_{t}=\left(\hat{\mathbf{u}}_{t}, \hat{\mathbf{s}}_{t}\right)$ can be then obtained either using the Maximum A Posteriori (MAP) or the Minimum Mean Square Error (MMSE) criterion. However, closed-form computations are not possible in the general case, therefore sub-optimal solutions are used instead, such as grid-based filters or PF. In this paper, we adopt a PF approach since it can attain the same accuracy as a grid-based one with less computational complexity. In this specific case, each particle $\mathbf{x}_{t}^{(n)}$ with $n=1, \ldots, N_{\mathrm{P}}$ is identified by the joint position-sight state $\left(\mathbf{u}_{t}^{(n)}, \mathbf{s}_{t}^{(n)}\right)$, with $\mathbf{u}_{t}^{(n)} \in \mathcal{U}$ and $\mathbf{s}_{t}^{(n)} \in \mathcal{S}$. For any additional information on the $\mathrm{PF}$ implementation of the tracking filter used to estimate $\mathbf{x}_{t}$, the interested reader can refer to Appendix A.

\section{Illustrative Example of the Proposed Method}

In this section, we provide a visual and intuitive representation of the proposed mixed LOS/NLOS model. In Fig. 2, we depict a snapshot of the TDoA measurement likelihoods and their combination in the factory scenario with link 2 between the tag and AP2 affected by NLOS with an excess range $\Delta d_{t}^{(2)}=-2 \mathrm{~m}$. The parameters used for the computation of the likelihoods are: $\sigma_{\mathrm{LOS}}=0.25 \mathrm{~m}, \sigma_{\mathrm{NLOS}}=0.5 \mathrm{~m}$ and $\sigma_{d}=2.5 \mathrm{~m}$. The TDoA measurements are computed by using AP1 as master anchor. For visualization purposes, the likelihoods have been independently rescaled to fit in the
TABLE II

Devices Technical Parameters

\begin{tabular}{cccc}
\hline & Decawave & Sewio & Ubisense \\
\hline Frequencies & $3.5-6.5 \mathrm{GHz}$ & $3-7 \mathrm{GHz}$ & $6-8 \mathrm{GHz}$ \\
Bandwidth & $500,900 \mathrm{MHz}$ & $500 \mathrm{MHz}$ & $800 \mathrm{MHz}$ \\
Measurement & ToA & TDoA & TDoA+AoA \\
Accuracy & $30 \mathrm{~cm}$ & $30 \mathrm{~cm}$ & $15 \mathrm{~cm}$ \\
Coverage & $290 \mathrm{~m}$ & $50 \mathrm{~m}$ & $160 \mathrm{~m}$ \\
\hline
\end{tabular}

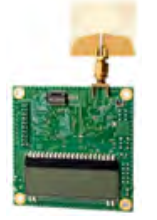

(a)

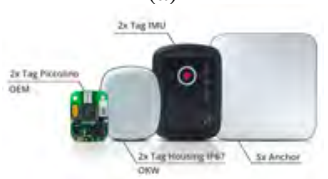

(b)

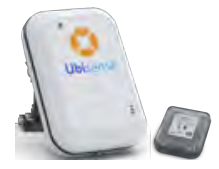

(c)

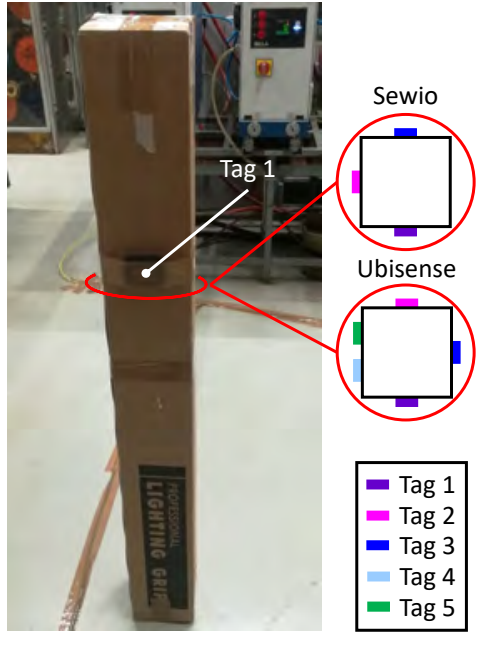

(d)
Fig. 3. UWB experimental setting: a) TREK1000 unit, b) Sewio RTLS TDoA kit, c) Ubisense Dimension4 sensor (left) and tag (right), d) cardboard box used for the experiments.

range $(0,1)$. Looking at the figure, it is intuitive to infer that if NLOS propagation is not compensated (i.e., all the the marginal likelihoods are computed using (10)), it exists a strong bias in the likelihood of $\rho_{t}^{(1,2)}$, which results to be translated far from the true tag position. This leads to an inaccurate localization in case of equally weighted product among all the three marginal pdfs. On the other hand, with the proposed NLOS mitigation, we have an enlarged marginal for $\rho_{t}^{(1,2)}$ which accounts for the NLOS bias statistics of the considered scenario (according to (15)) and provides more accurate localization once all the likelihoods are multiplied together: i.e., the product is very close to the true position as the NLOS range offset is compensated.

\section{UWB TEChNOLOGIES FOR TESTING}

This section presents an overview of the UWB technologies selected for testing: Decawave TREK1000 (Fig. 3a), Sewio RTLS TDoA kit (Fig. 3b) and Ubisense Dimension 4 (Fig. 3c). Table II summarizes their main specifications.

\section{A. Decawave}

The Decawave TREK1000 evaluation kit is composed of 4 UWB units. Each board, depicted in Fig. 3a, is equipped with a DW1000 module, an STM32F105 ARM Cortex M3 processor and an omnidirectional antenna. The DW1000 module is an UWB ranging chip compliant to the IEEE 802.15.4-2011 UWB standard [65]. It spans six Radio Frequency (RF) bands 
in the frequency range $3.5-6.5 \mathrm{GHz}$, with a bandwidth of $500 \mathrm{MHz}$ or $900 \mathrm{MHz}$ and three different transmission rates: $110 \mathrm{kbit} / \mathrm{sec}, 850 \mathrm{kbit} / \mathrm{sec}$ and $6.8 \mathrm{Mbit} / \mathrm{sec}$. It is fully configurable to support two-way ranging via Time of Flight (ToF) estimation or one-way TDoA. Synchronization among reference nodes is performed wirelessly, allowing an easy installation and preventing from laborious prior calibration. The reported ranging accuracy is $10 \mathrm{~cm}$, which translates into $30 \mathrm{~cm}$ accuracy for $3 \mathrm{D}$ positioning. The maximum coverage supported is $290 \mathrm{~m}$. The TREK1000 kit supports only two channels, namely the second and fifth centered around 4 and $6.5 \mathrm{GHz}$, respectively, and two data rates: $110 \mathrm{kbit} / \mathrm{sec}$ and $6.8 \mathrm{Mbit} / \mathrm{sec}$. Using the dip-switches provided on the board, it is possible to configure each unit as tag or anchor and choose the channel specifications. The location update rate provided by each board is $3.57 \mathrm{~Hz}$.

\section{B. Sewio}

Sewio products make use of the Decawave DW1000 UWB ranging chip to provide location estimates based on TDoA measurements. The manufacturer reports an accuracy of 30 $\mathrm{cm}$ considering 3D localization with a maximum coverage of $50 \mathrm{~m}$. According to the documentation, the kit tested herein does not employs any tracking filtering, but it has been made available in the new model version, the RTLS UWB kit. Sewio ships the RTLS TDoA kit depicted in Fig. 3b, which is composed of five anchors, two IMU tags, two Piccolino tags and the license for the RTLS Studio Platform and SAGE Analytics, two software packages used for configuration, management and data visualization purposes. Anchors are reference nodes equipped with the DW1000 chip and an omnidirectional antenna. Rather than relying on timing cables, anchors' are synchronized wirelessly. This allows the system to be configured quickly, making it suitable for any environment. Tags employ the same chip, an omnidirectional antenna, an ultra-low-power ARM EFM32G M3 (for Piccolino tags) and inertial sensors. The IMU tag supports all available sensors (i.e. accelerometer, gyroscope, magnetometer, barometer, thermometer) while Piccolino only the accelerometer. The tags have preconfigured update rates of $1 \mathrm{~Hz}$ and $10 \mathrm{~Hz}$ in case of static and dynamic conditions, respectively.

\section{Ubisense}

We use the UWB system Ubisense Dimension4 (D4), which combines TDoA and AoA measurements to estimate the tag's position by Bayesian filtering. The manufacturer reports that only two anchors are sufficient for 3D location estimation. The UWB pulses are transmitted at a central frequency of $6.55 \mathrm{GHz}$, occupying a bandwidth of $800 \mathrm{MHz}$. The nominal accuracy is $15 \mathrm{~cm}$ in LOS conditions, while for complex environments it reduces to $50 \mathrm{~cm}$. The maximum coverage is $160 \mathrm{~m}$. The research and development kit is composed by four sensors, ten tags and the license for SmartSpace platform, a configuration and data visualization utility. Each sensor, depicted in Fig. 3c (left), is an anchor node that hosts an antenna array, the UWB radio receivers, a built-in accelerometer, and a processor. Sensors' synchronization is

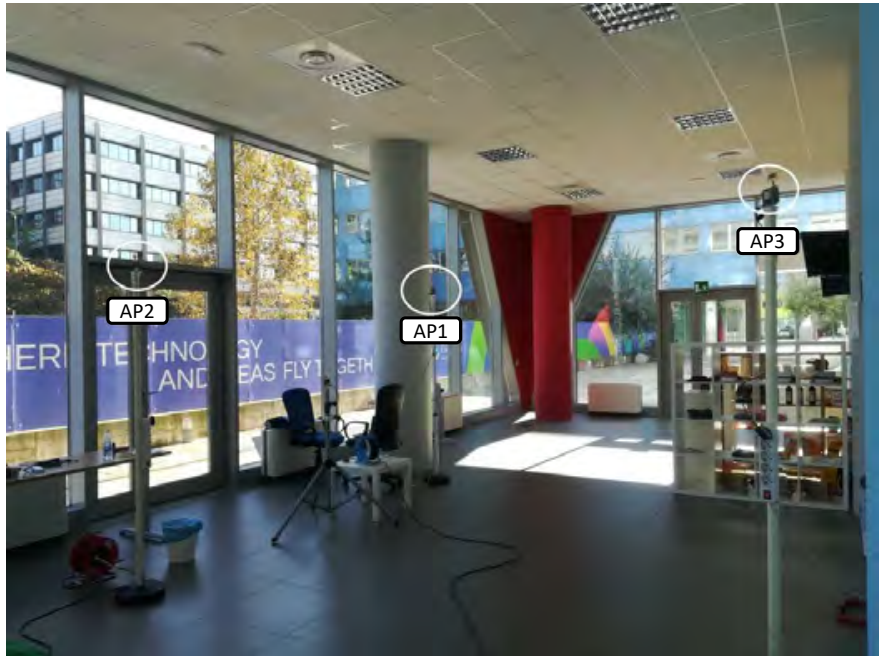

Fig. 4. UWB localization in indoor environment at IoTLab - Politecnico di Milano: test area and anchors' deployment.

performed via timing cables. On the other hand, tags, shown in Fig. 3c (right), are ultra-low power UWB transmitters, equipped with a motion detector and a battery. They support two main location update rates, namely the slow and the fast rates, that can be configured according to the specific application (the maximum update rate can be increased up to $30 \mathrm{~Hz}$ ).

\section{EXPERIMENTAL TESTS IN INDOOR SCENARIOS}

This section presents the experimental tests carried out to assess the performances of the UWB systems presented in Sec. III. We first analyze the capabilities of Decawave UWB localization kit in laboratory facilities (Sec. IV-A), then we analyze the results of experiments carried out in the Pirelli Tyre S.p.A. research plant (Sec. IV-B). Due to project requirements and industrial constraints, Ubisense and Sewio were both tested in the research plant while only Decawave was available in the laboratory. The goal of the laboratory tests is to evaluate the separate impact of possible impairments that arise in indoor environments under controlled conditions, while the experimental tests are aimed at assessing the overall localization performances of UWB systems in real operating industrial environments. Moreover, the selection of Ubisense and Sewio devices allows us to collect raw data on TDoA and AoA for the evaluation of the proposed tracking methodology. The analysis aims to assess the capabilities of UWB indoor localization in terms of both the location error and the accuracy of the raw range/angle radio measurements used for localization. We evaluate the location error as $\varepsilon_{t}=\left\|\hat{\mathbf{u}}_{t}-\mathbf{u}_{t}\right\|$, the ranging error $\varepsilon_{r, t}=\rho_{t}-d_{t}$, the azimuth error $\varepsilon_{\alpha, t}=\rho_{\alpha, t}-\alpha_{t}$ and the elevation error $\varepsilon_{\beta, t}=\rho_{\beta, t}-\beta_{t}$, computed over all the $L$ APs. To simplify the notation, from now on the temporal subscript $t$ on the errors is removed as it should be easily inferred from the discussion on each specific analysis.

\section{A. Laboratory Scenario}

A preliminary validation of the UWB technology for positioning has been carried out in the laboratory facility IoTLab 


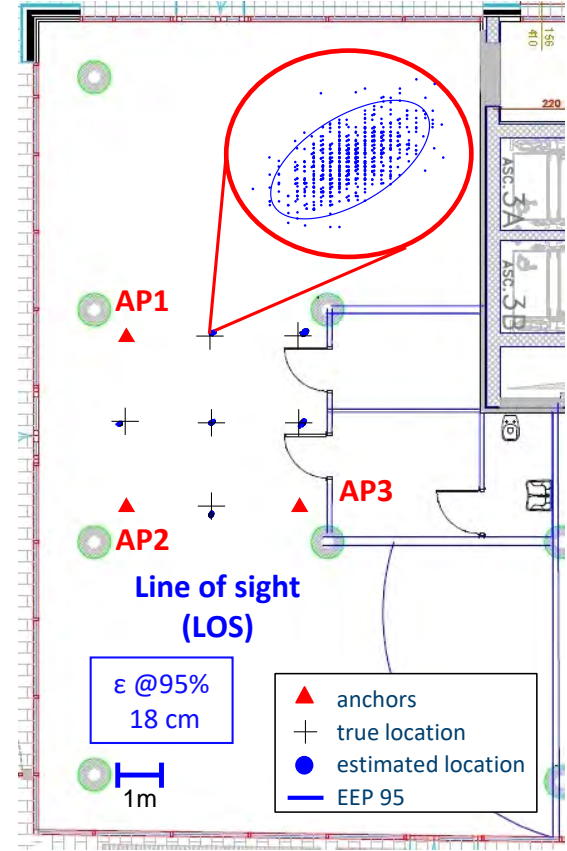

(a) LOS

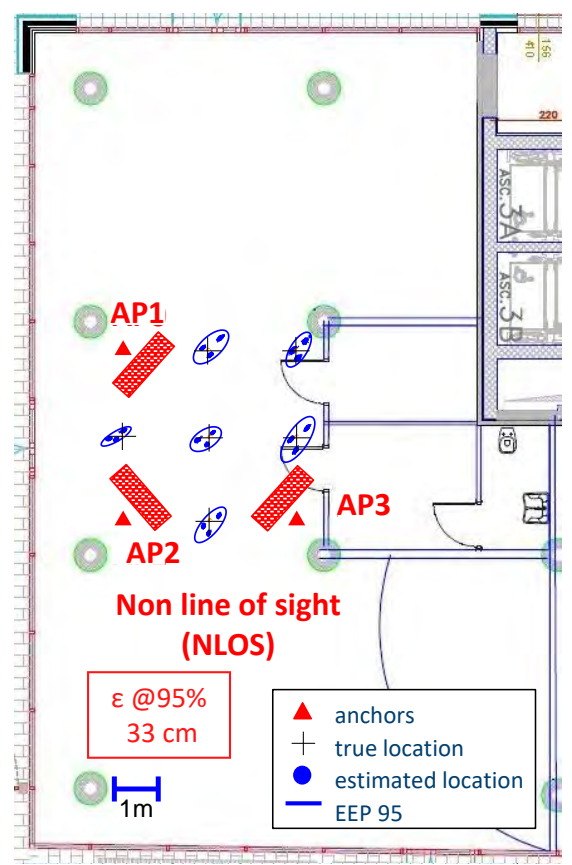

(b) NLOS

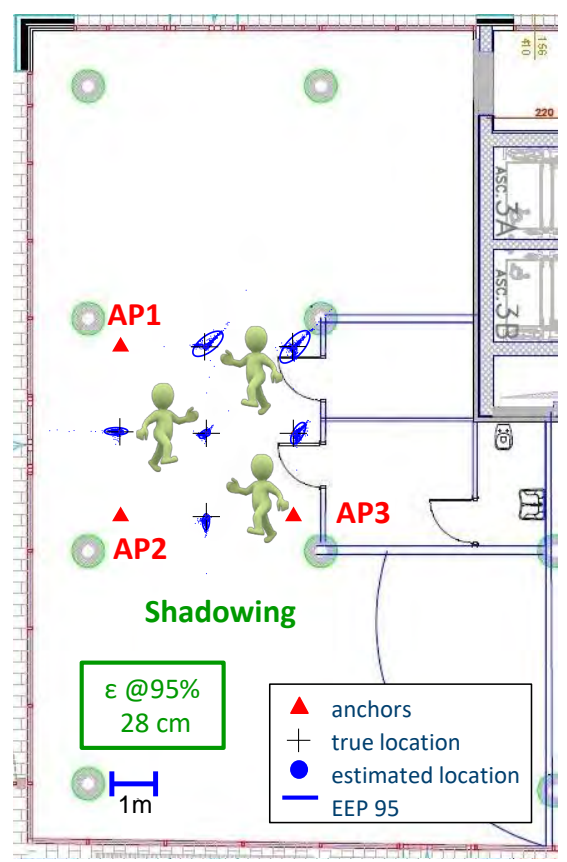

(c) Shadowing

Fig. 5. UWB localization in an indoor environment at IoTLab - Politecnico di Milano for different propagating conditions: a) LOS, b) NLOS c) shadowing. By fixing the reference system as coincident with AP1, i.e., $\mathbf{p}_{1}=(0,0) \mathrm{m}$, with $\mathrm{x}$ axis pointing downwards and y axis rightwards, the six positions selected for the experiments are: $(1.79,0) \mathrm{m},(0,1.79) \mathrm{m},(1.79,1.79) \mathrm{m},(3.57,1.79) \mathrm{m},(0,3.57) \mathrm{m},(1.79,3.57) \mathrm{m}$. Dimension of the area: $18 \times 11 \mathrm{~m}$.

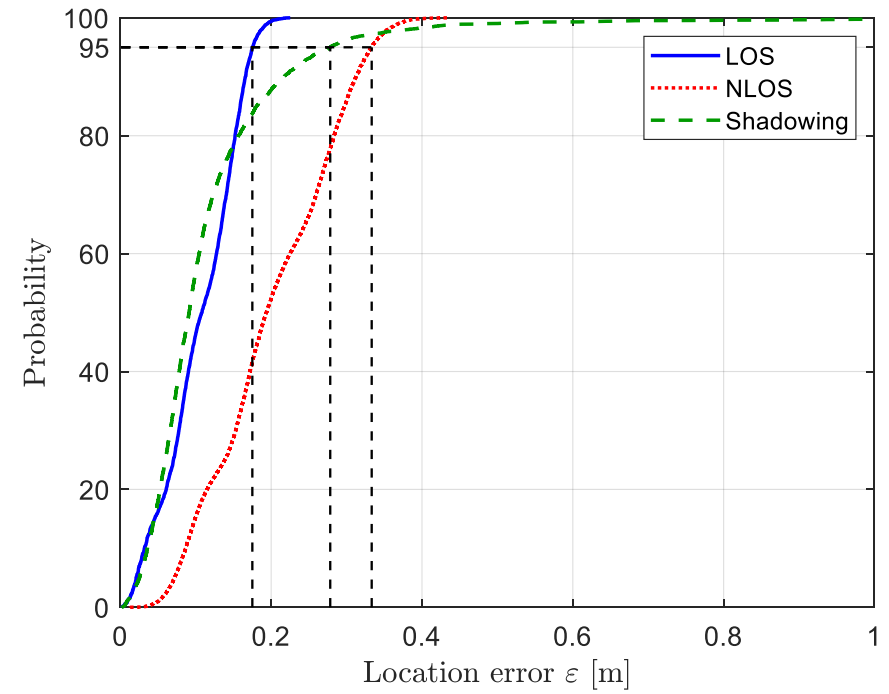

Fig. 6. CDF of UWB localization accuracy in indoor environment at IoT Lab - Politecnico di Milano for LOS, NLOS and shadowing conditions.

of Politecnico di Milano, shown in Fig. 4. We used Decawave TREK1000 devices to conduct a number of experiments in a controlled environment, evaluating how propagation conditions affect the localization performance.

In all the experiments, three APs are placed at fixed locations over a tripod at a height of $2 \mathrm{~m}$, as illustrated in Fig. 4. Six Ground-Truth (GT) positions, measured with a laser distance meter, are chosen to evaluate the performance of UWB static localization The selected positions are depicted by the cross markers in Fig. 5 and specified in the caption of the same figure. For each position, the tag is mounted on a tripod at a height of $1 \mathrm{~m}$ and UWB measurements are recorded for 240 seconds. The total number of location fixes for each position is 857 (i.e., $240 \mathrm{~s} \times 3.57 \mathrm{~Hz}$ ).

To characterize the main problems that arise in indoor environments and assess their impact on the localization accuracy, three experimental tests performed under different propagation conditions are presented: LOS, NLOS and shadowing propagation. A non obstructing link between anchors and tag is considered in the LOS experiment. On the other hand, for the NLOS test, metallic shields are placed in front of the anchors. The metallic shield employed in the test is square-shaped with dimensions $0.4 \times 0.4 \mathrm{~m}$. Finally, shadowing propagation is simulated by making three people move around the tags, following a circular trajectory.

Fig. 5 shows the location estimates provided by the TREK1000 software as black dots, the true positions as cross symbols, and the Elliptical Error Probable at 95\% confidence (EEP95) confidence as the black solid line for the aforementioned experiments. The LOS test (Fig. 5a) shows location estimates close to the real position. On the contrary, the NLOS (Fig. 5b) and the shadowing (Fig. 5c) ones exhibit a mismatch between true and estimated locations. The NLOS test shows a positive range bias whereas the shadowing one exhibits a large dispersion of the position estimates. It should be noted that, in the NLOS test, three clusters appear as a result of the experiment setup. The APs are separately obscured using the metallic shield one at a time, thus, each cluster originates from a particular AP affected by NLOS. On the other hand, the shadowing test presents a wider position dispersion (without the distinction of the three separate clusters) compared to 




(a) LOS

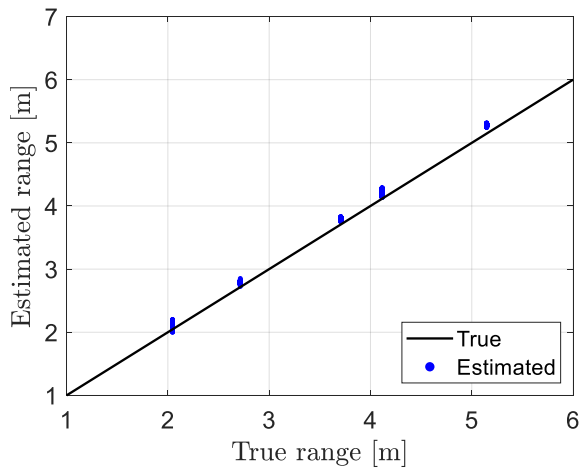

(d) LOS

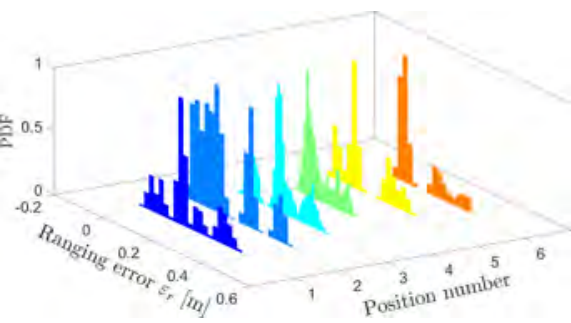

(b) NLOS



(e) NLOS

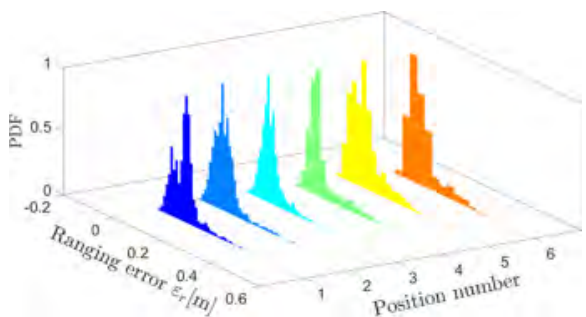

(c) Shadowing

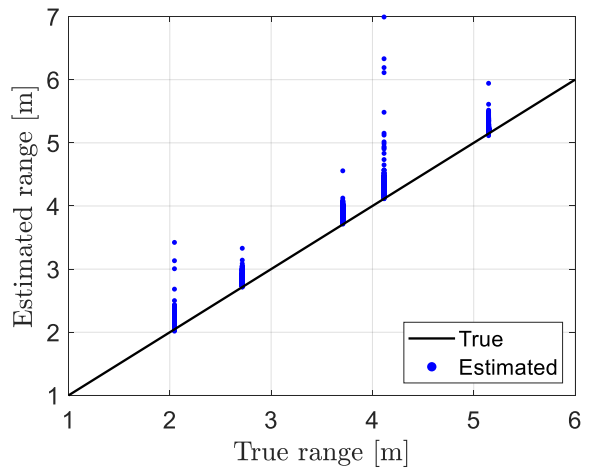

(f) Shadowing

Fig. 7. Decawave UWB ranging statistics: a) LOS, b) NLOS and c) Shadowing histograms for each position, d) LOS, e) NLOS and f) Shadowing real vs estimated ranges for aggregated data.

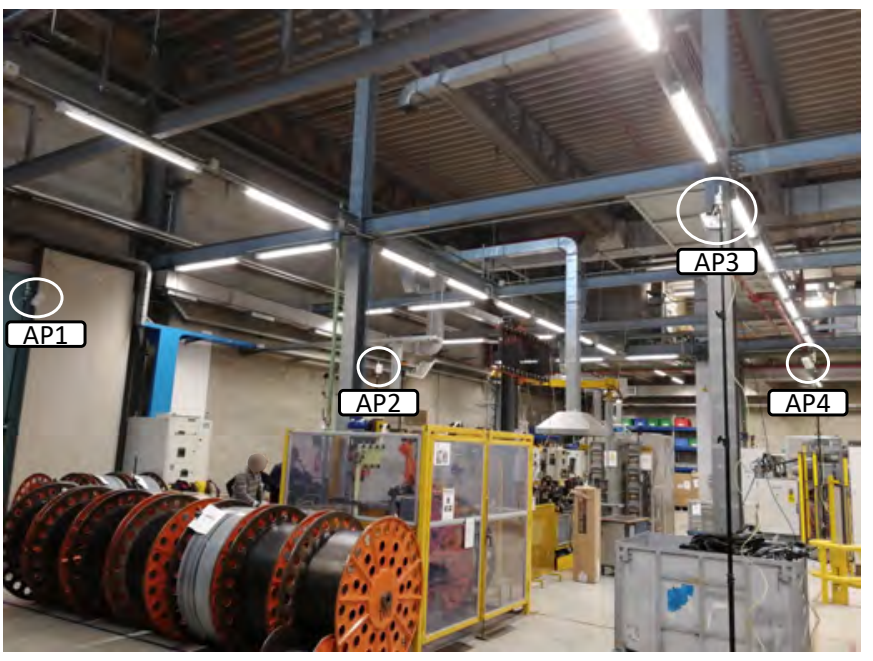

Fig. 8. UWB localization in industrial environment at Pirelli Tyre S.p.A. research plant: tracking area and anchors' deployment.

the NLOS one, resulting from the presence of walking people that generate random propagation conditions (shadowing, scattering and diffraction) for each tag-AP link. Rather than completely blocking the UWB signal as the metallic shield does, the main impact of shadowing is a random fluctuation of the power of the first signal arrival at the AP, leading to errors in the detection of the signal peak.

A more detailed analysis of these estimates is provided in Fig. 6, where the Cumulative Distribution Function (CDF) of the location error $\varepsilon$ of aggregated data is reported for all three tests. In $95 \%$ of the occurrences, the LOS test attains a positioning error lower than $18 \mathrm{~cm}$, while $28 \mathrm{~cm}$ and $33 \mathrm{~cm}$ are achieved in the NLOS and shadowing cases.

The last analysis on the positioning accuracy of Decawave system is presented in Fig. 7. The upper part of the figure reports the histogram of the ranging error $\varepsilon_{r}$ for each position considered in the experiments, while the bottom part highlights the relationship between true and estimated ranges aggregated over all positions. Comparing the results, the LOS test shows ranging errors that are distributed around zero with a maximum value of $0.22 \mathrm{~m}$ for all positions. On the other hand, the NLOS and shadowing tests present far more dispersion resulting in long-tail behaviours. More specifically, in case of NLOS, the error distribution presents a second component centered around $0.3 \mathrm{~m}$ due to the blockage of the direct path, while the shadowing one exhibits a decaying tail that extends up to $0.5 \mathrm{~m}$ caused by the rapidly varying received power. Considering the combined effects caused by NLOS and shadowing propagation, high accuracy localization may be difficult to achieve, especially if the impairments are not compensated properly. This is further exacerbated when considering highly complex scenarios, such as industrial environments, as discussed in the next section.

\section{B. Industrial Scenario}

In this section, we present the second set of tests carried out in an industrial site of Pirelli Tyre S.p.A. (see Fig. 8), where Ubisense and Sewio devices are validated. Both static localization as well as dynamic location tracking are considered in the experimental tests.

As depicted in Fig. 8, four APs are deployed in the site at a height of $3.73 \mathrm{~m}$, both for Sewio and Ubisense. The UWB tags (three for Sewio, five for Ubisense) are attached onto a cardboard box (see Fig. 3d) and moved across 25 locations at a height of $1 \mathrm{~m}$. The positions selected for the industrial tests are reported in Fig. 9, where the spacing between each one of them 


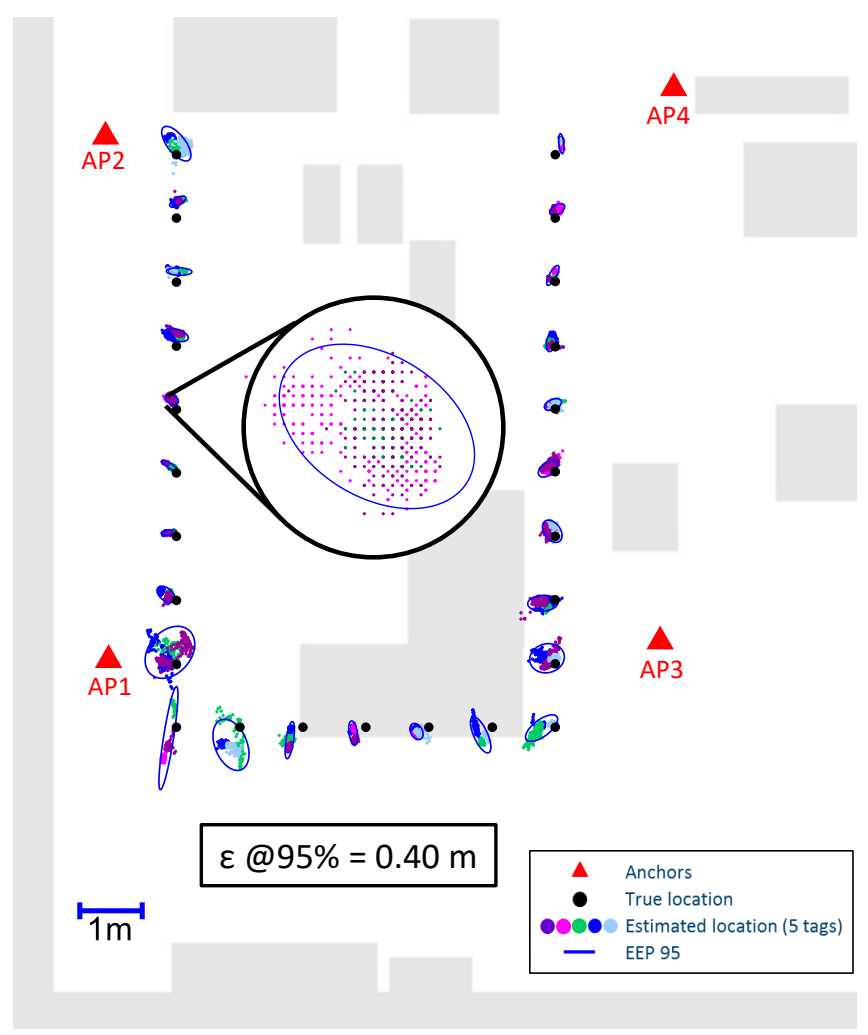

(a) Ubisense

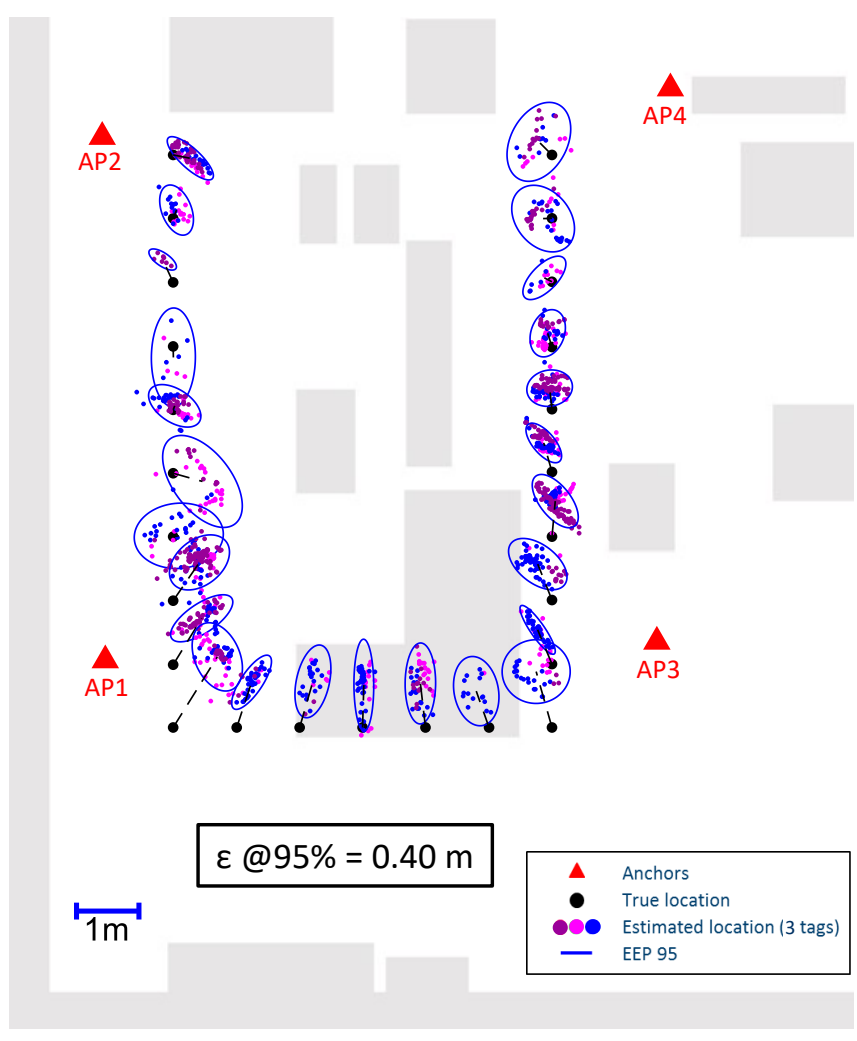

(b) Sewio

Fig. 9. UWB localization at Pirelli Tyre S.p.A. research plant - Static tags: a) Ubisense, b) Sewio. Light gray areas indicate walls, machines or cabinets.

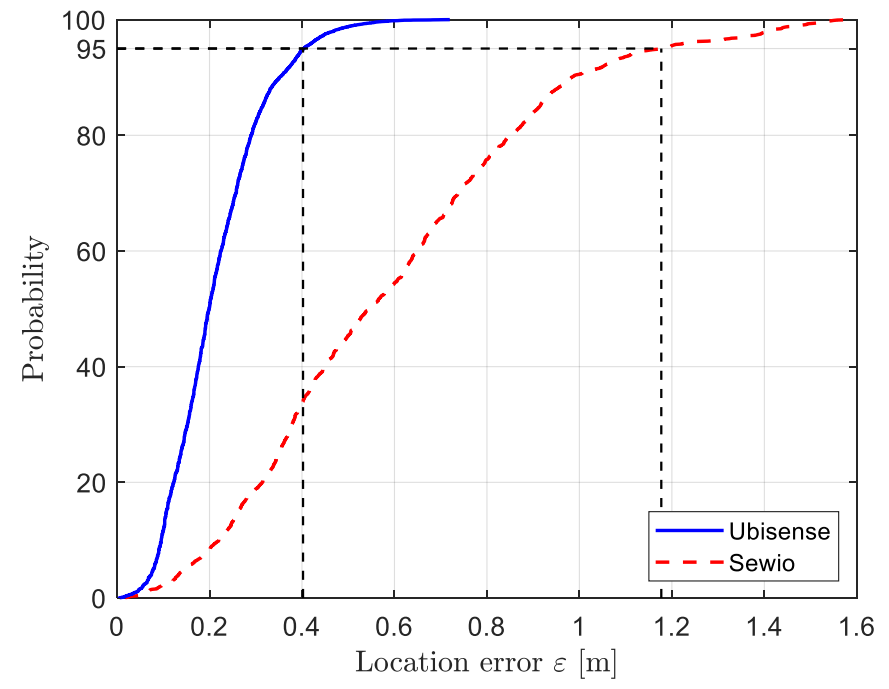

Fig. 10. CDF of UWB localization (static tags) in industrial scenario at Pirelli Tyre S.p.A. research plant for Ubisense and Sewio.

is $1 \mathrm{~m}$. In each location, thirty seconds of measurements are gathered while the tags are still in their positions. A transition between two adjacent locations is manually done while the system is running, therefore, to avoid mobility effects, we discard the three initial and three final seconds (out of thirty) for each location. The whole experiment takes $30 \times 25=750 \mathrm{~s}$ for completion, 600 of which are considered as valid. The measured update rates found throughout the test are $7.44 \mathrm{~Hz}$ for Ubisense and $1.48 \mathrm{~Hz}$ for Sewio, which corresponds to a total number of location estimates of 22320 and 2592, respectively.
The results of the UWB validation process are illustrated in Fig. 9 for both systems, where the location estimates provided by the two solutions translated to the cardboard box center are reported as colored dots, each one referring to a different tag, and the EEP95 is superposed using the solid black line. To highlight the position biases, we added a dashed line that connects the mean location estimate to each respective true position. The comparison between the two technologies shows that Ubisense provides a higher accuracy than Sewio in the considered setting. This was expected as Ubisense uses both TDoA and AoA for estimating the position (Sewio only TDoA), it employs Bayesian filtering (as opposed to no filtering), and has a larger bandwidth of $800 \mathrm{MHz}$ (compared to $500 \mathrm{MHz}$ ). Nevertheless, a performance degradation for both systems is experienced in the lowest part of the layout (i.e., between AP1 and AP3), where severe propagating conditions are encountered due to metallic objects that obscure the UWB tags. Furthermore, this portion of the map is outside the area delimited by the anchors, that negatively impact the geometric performance factor.

The analysis on the overall localization performance in terms of CDF of the positioning error is provided in Fig. 10. In $95 \%$ of the case Ubisense achieves a localization error $\varepsilon$ less then $40 \mathrm{~cm}$, while Sewio attains a larger value of $1.17 \mathrm{~m}$. Results indicate that the optimized combination of TDoA and AoA, jointly with the employment of Bayesian filtering techniques, as performed by Ubisense, outperforms stand-alone TDoA-based systems. Nevertheless, the calibration process demanded by Ubisense might not be always 




(a) TDoA

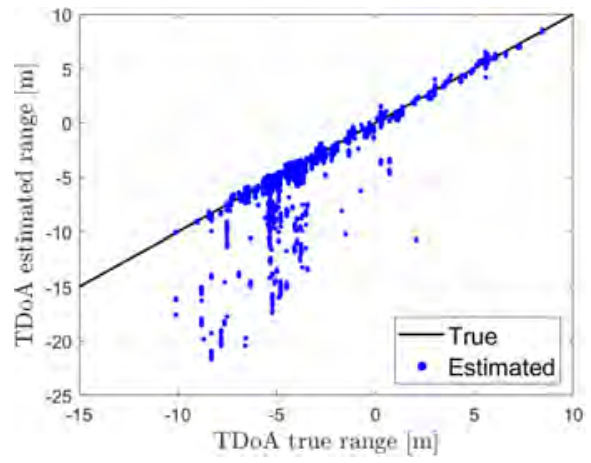

(d) TDoA

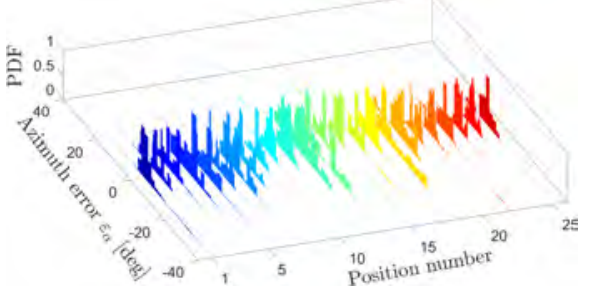

(b) Azimuth

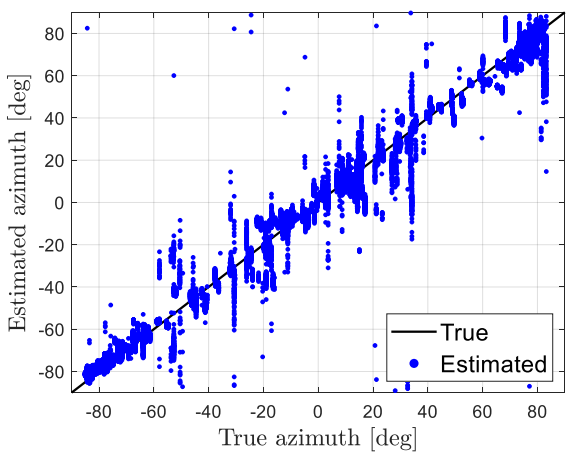

(e) Azimuth

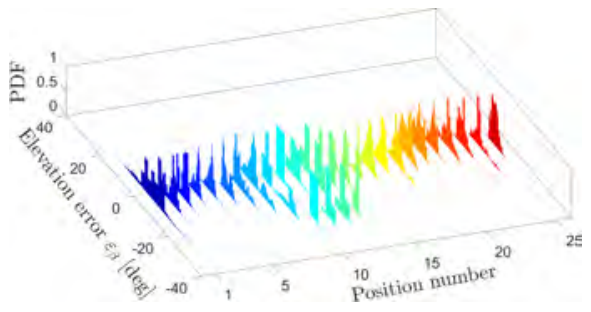

(c) Elevation

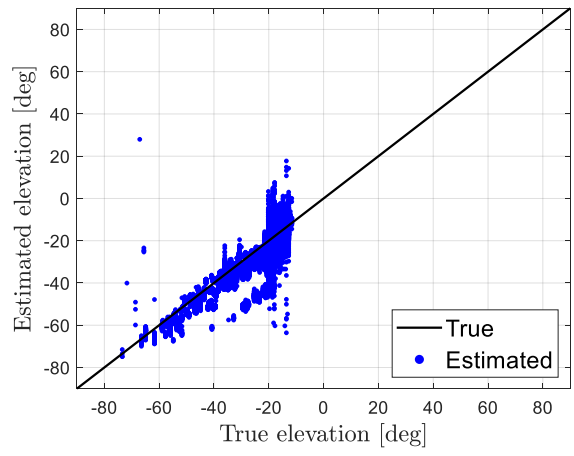

(f) Elevation

Fig. 11. Ubisense UWB localization statistics: a) TDoA, b) Azimuth and c) Elevation histograms for each position of the trajectory, d) TDoA, e) Azimuth and e) Elevation real vs estimated measurements aggregated over all positions.

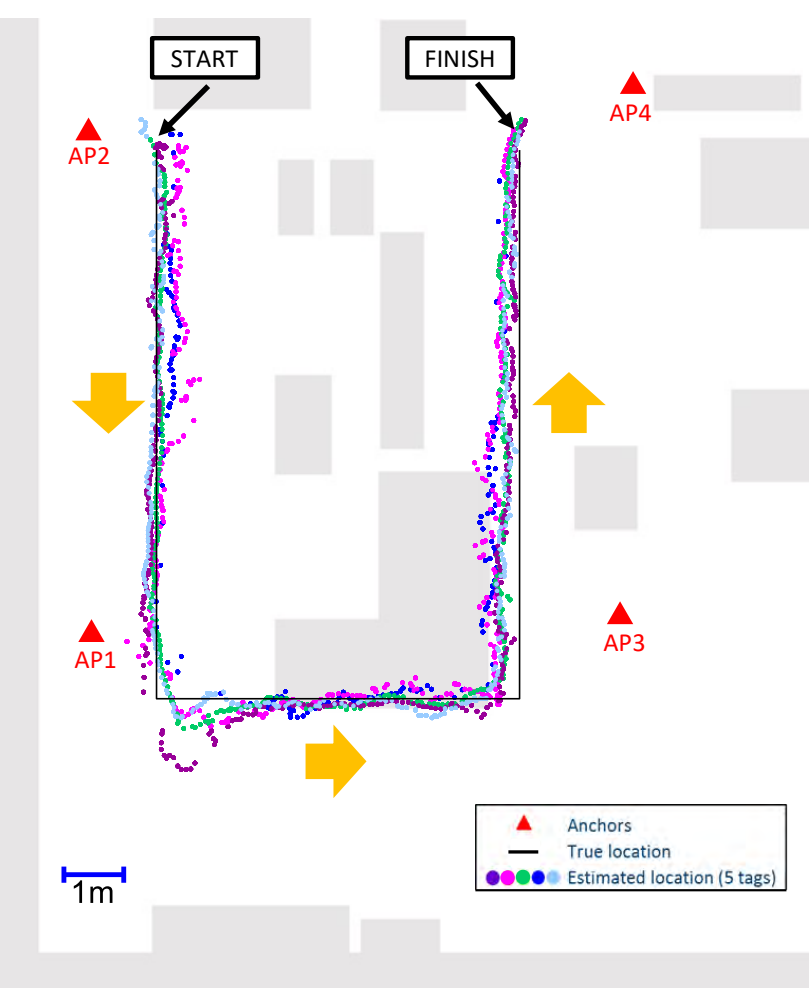

(a) Ubisense

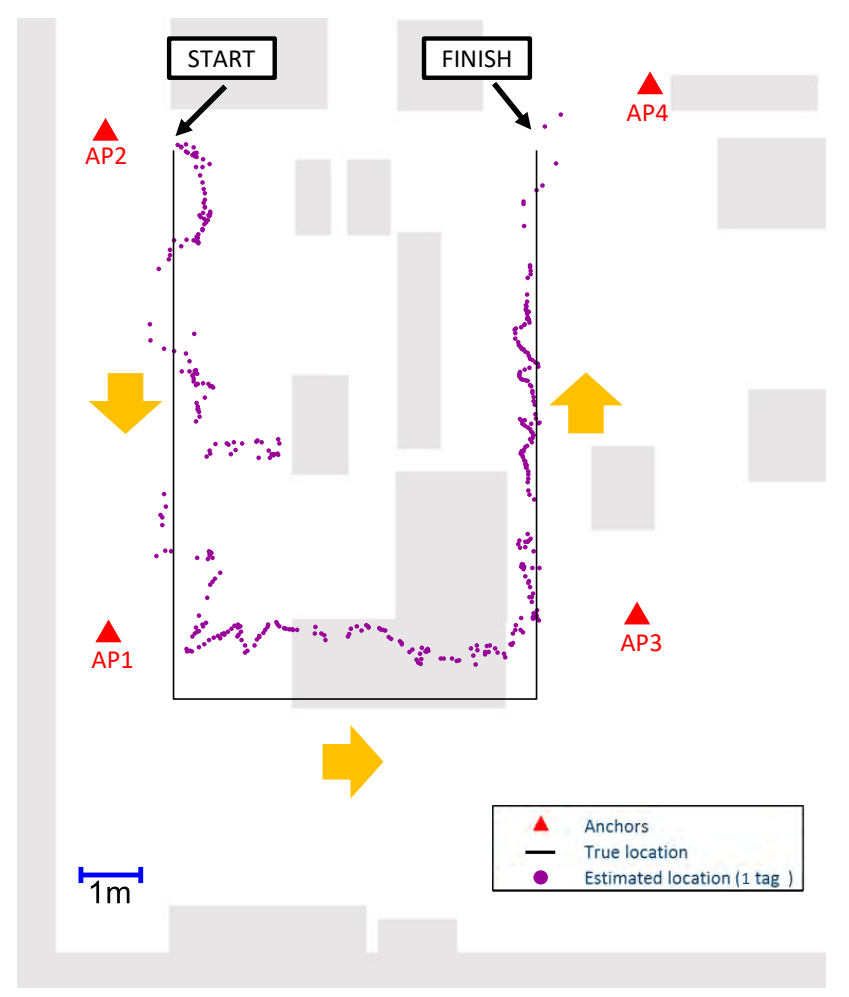

(b) Sewio

Fig. 12. UWB localization at Pirelli Tyre S.p.A. research plant - Mobile tags: a) Ubisense, b) Sewio. Light gray areas indicate walls, machines or cabinets.

feasible in industrial applications. Conversely, Sewio requires less infrastructure installation and calibration effort.

Similarly to the analysis in Fig. 7 for the laboratory scenario, here we investigate the accuracy of raw UWB measurements to assess the impact of the industrial environment on the ranging performance. We opted to analyze the raw TDoA and AoA data for Ubisense as these data were not accessible for Sewio. For TDoA, the measurements are referred to a master anchor (i.e, $\ell$-th AP in Sec. II) that is dynamically selected at each time step based on the received power so as to guarantee that it is as much as possible in LOS condition. On the other hand, AoAs are reported as low-level fields in the logfile. Not all 


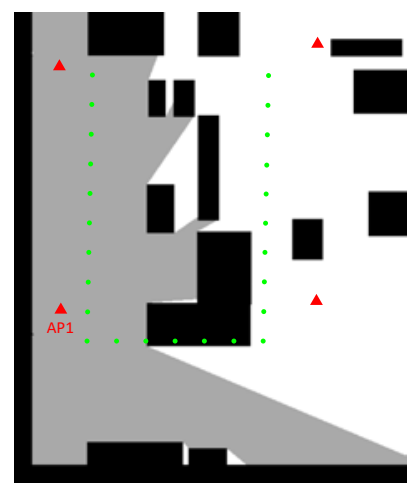

(a) AP1

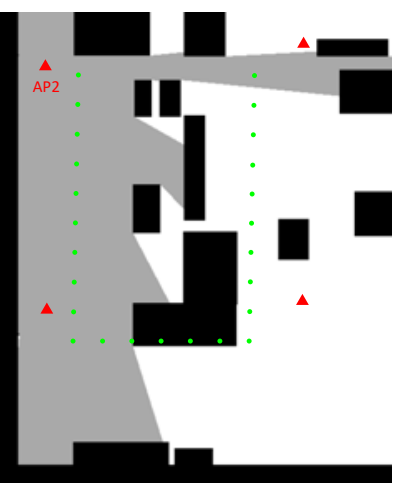

(b) AP2

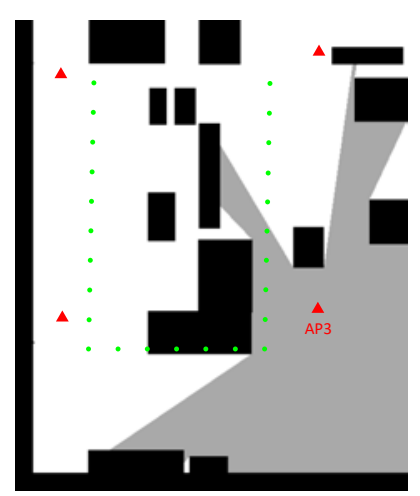

(c) AP3

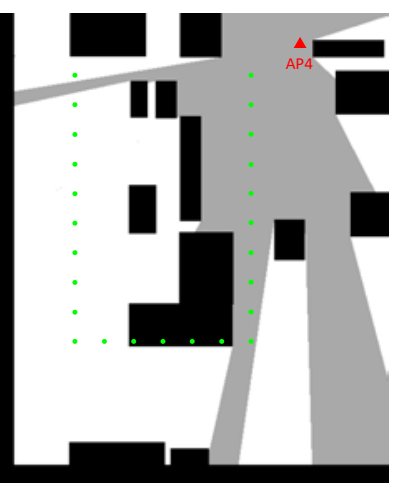

(d) AP4
Fig. 13. LOS/NLOS Coverage Maps: a) AP1, b) AP2, c) AP3, and d) AP4. Gray areas indicate LOS condition, white areas imply NLOS and black areas denote walls, machines or cabinets.

anchors were available at each time instant, due to poor signal quality. Nevertheless, we assured to gather as much raw data as to guarantee a reliable statistical characterization.

Fig. 11 reports the histogram of the UWB measurement errors for each position of the considered trajectory (top figure), together with the plots of the measured vs. true location-related parameters aggregated over all locations (bottom figure). Note that the TDoA ranging error is computed as the difference between the ranging error of the master AP and the ranging error of each other AP. In the TDoA case (Figs. 11a and 11d), the measurements are distributed around the true TDoA for most of the positions. However, from position 3 to 9 and from 11 to 17 , long tail distributions are detected which indicate that non-Gaussian behavior is experienced as a result of multipath. Azimuth and elevation AoAs (Figs. 11b-11e and Figs. 11c$11 \mathrm{f}$, respectively) present a wide angular dispersion up to more than $20 \mathrm{deg}$. In particular, for position 11, 12 and 17, the azimuth AoA is quite spread, indicating the presence of metallic scatterers that lower the accuracy in angle estimation. This behaviour has been detected also for the elevation data, where large errors are detected from position 8 to 13 .

The last analysis is dedicated to a dynamic localization test where the tags were moved along the "U" walk, crossing the 25 GT positions of Fig. 9. Fig. 12 shows the trajectory estimated by Ubisense (Fig. 12a) and Sewio (Fig. 12b) (colored dots correspond to different tags). Again, Ubisense outperforms Sewio, confirming that the combination of hy- brid (TDoA and AoA) measurements by tracking filters is mandatory for high precision positioning in harsh industrial environments. Nevertheless, NLOS mitigation techniques are required for dealing with severe propagating conditions, as in the lowest portion of the scenario, where false localization is highlighted.

\section{AnAlysis of the JMS Filter PerformanCE}

This section is dedicated to the validation of the Bayesian tracking filter presented in Sec. II. The numerical assessment of the proposed technique is performed using the raw TDoA and azimuth AoA data extracted from the Ubisense system by the tests in Sec. IV. The results of the proposed technique are presented for the TDoA+AoA (azimuth) case and TDoAonly. The comparison is performed considering two baseline approaches: the first one compensate the NLOS propagation by employing a univariate Skew $\mathrm{t}$ distribution, as reported in previous state-of-the-art methods [36], [37], while the second one does not employ any compensation technique. We first detail the setting parameters and configurations in Sec. V-A, then the results of tracking are reported in Sec. V-B.

\section{A. System Parameters and Configuration}

The performance of the proposed augmentation method is evaluated in the same industrial test as in Sec. IV-B. Due to the way each TDoA is delivered by Ubisense, only 2 out of 4 conditioned probabilities of the model described in Sec. II-A are experienced. In fact, since the master anchor for multi-lateration is selected as the one with the most favorable propagation conditions (i.e., close to LOS), the observed sight states reduce to LOS/LOS (i.e., $s_{t}^{(\ell)}=0, s_{t}^{(k)}=0$ ) and LOS/NLOS (i.e., $s_{t}^{(\ell)}=0, s_{t}^{(k)}=1$ ). This behavior has been found analyzing all the available raw data extracted by Ubisense software. On the other hand, all the available azimuth AoA are processed and aggregated together to form the overall dataset. The collected data covers $N_{\mathrm{TF}}=25$ realizations of trajectory of 25 positions shown in Fig. 9, with $N=8$ TDoA and azimuth AoA measurements collected by each AP in each position over $1 \mathrm{~s}$, corresponding to approximately 200 measurements. The likelihood of each set of $N$ measurements is obtained by multiplying the corresponding $N$ marginal likelihoods.

As far as the sight evolution process is concerned, we rely on the floor plan of the area to extract the sight condition of all $L$ APs and build the LOS/NLOS maps shown in Fig. 13. The coverage maps are computed by performing ray-tracing from each tag location $\mathbf{u}_{t} \in \mathcal{U}$ (excluded the black areas, which are walls, machines or cabinets) to the position $\mathbf{p}_{\ell}$ of each AP. For each tag-AP link $\ell$, the sight condition $s_{t}^{(\ell)}$ is selected to be in LOS (i.e., $s_{t}^{(\ell)}=0$ ) if the line connecting the tag position $\mathbf{u}_{t}$ and the AP position $\mathbf{p}_{\ell}$ does not intersect any black point, otherwise $s_{t}^{(\ell)}=1$ (i.e., NLOS). Using this technique, the sight processes are deterministically assigned by the geometry layout, relaxing the independence assumption between positions and sight states. This procedure may not be fully representative of the actual wireless propagating conditions since it does not include any dynamic changing 


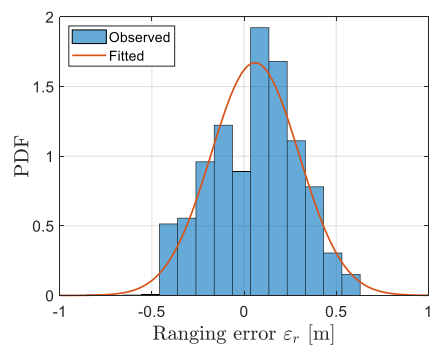

(a) LOS/LOS TDoA



(b) LOS/NLOS TDoA

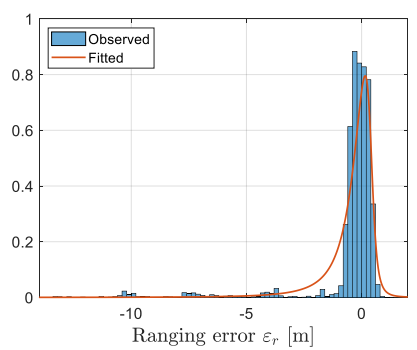

(c) Aggregated TDoA

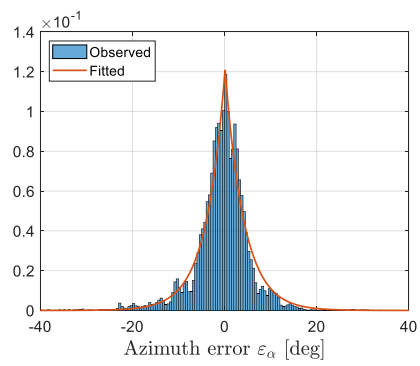

(d) Aggregated Azimuth AoA

Fig. 14. Comparison of observed statistics with respect to fitted data: a) LOS/LOS TDoA, b) LOS/NLOS TDoA, c) Aggregated TDoA d) Aggregated azimuth AoA. For each histogram is superimposed with the solid red line the fitted distribution: a) Gaussian, b) EMG, c) Skew t, d) Laplace.

of the environment and it does not consider the vertical axis, leading to possible misclassifications of sight conditions. Nevertheless, the proposed approach can be extended to 3D maps when available and periodically re-trained to account for possible changes.

To calibrate the sight evolution probabilities $\left\{p_{0}, p_{1}\right\}$, we simulate a training trajectory of length $\mathrm{I}=10^{4}$ samples in the layout. The relative frequencies of transition from LOS to LOS $\left(p_{0}\right)$ and from NLOS to LOS $\left(p_{1}\right)$ are evaluated by counting how many transitions occur within the training trajectory for all APs yielding $p_{0} \approx p_{1} \approx 0.8$.

A statistical characterization of UWB measurements is given in Fig. 14, where the histogram of TDoA and azimuth AoA errors are shown. For TDoA, the analysis considers both the separate cases of LOS/LOS and LOS/NLOS conditions, in Fig. 14a and Fig. 14b, respectively, as well as the aggregated TDoA in Fig. 14c. For azimuth AoA, instead, the histogram of aggregated data is illustrated in Fig. 14d. On top of the histograms in Fig. 14, a red curve of associated fitted distribution is superimposed. Specifically, we have that the separate cases of LOS/LOS and LOS/NLOS are modeled as discussed in Sec. II-B, leading to the Gaussian and EMG pdfs in (10) and (15), respectively, with fitting parameters summarized in Table III. Without distinguishing between the two conditions, we also consider a state-of-the-art model of the aggregated TDoA that employs a Skew t distribution [36], [37], i.e., $p\left(\rho_{t}^{(\ell, k)} \mid \mathbf{u}_{t}\right)=S T\left(\rho_{t}^{(\ell, k)} ; d_{t}^{(\ell, k)}+\mu_{s t}, \sigma_{s t}^{2}, \delta_{s t}, \nu\right)$. In this case, the fitting yields to the following parameters: $\mu_{s t}=-0.421 \mathrm{~m}, \sigma_{s t}=0.224 \mathrm{~m}, \delta_{s t}=0.652 \mathrm{~m}, \nu=4$. Lastly, the azimuth AoAs follow a Laplace distribution with diversity parameter $b_{\alpha}=4.13 \mathrm{deg}$.

We would like to remark that, as an alternative to our approach, $\sigma_{d}$ can also be chosen according to the tabulated values reported in experimental studies on UWB channel modeling such as in [54], [66]-[68]. Typical values range from 1 up to $60 \mathrm{~ns}$ and are ruled by the specific environment considered. The value found fitting the delay distribution is $1.43 \mathrm{~ns}$ (i.e., $0.43 / c$, where $c$ is the speed of light), which is consistent with the literature. As a second comment, to cope with the heavy tail in the histogram in Fig. 14b, we added a constant term to the EMG pdf, corresponding to $3 \%$ of the model's peak. Lastly, regarding the azimuth AoA, our fitting lead us to select $b_{\alpha}=4.13 \mathrm{deg}$, even if higher values in the range $5-27$
TABLE III

FITTING PARAMETERS FOR THE PROPOSED LOS/NLOS MODELING.

\begin{tabular}{cc|cc}
\hline parameter & value & parameter & value \\
\hline$\sigma_{\mathrm{LOS}}$ & $0.169 \mathrm{~m}$ & $\sigma_{\mathrm{NLOS}}$ & $0.16 \mathrm{~m}$ \\
$\sigma_{1}=\sqrt{2} \sigma_{\mathrm{LOS}}$ & $0.239 \mathrm{~m}$ & $\sigma_{2}=\sqrt{\sigma_{\mathrm{LOS}}^{2}+\sigma_{\mathrm{NLOS}}^{2}}$ & $0.232 \mathrm{~m}$ \\
$\sigma_{d}$ & $0.43 \mathrm{~m}$ & $\mu_{n}$ & $0.328 \mathrm{~m}$ \\
\hline
\end{tabular}

deg can be experienced in indoor environments [56], [59][61]. However, we recall that Ubisense outputs filtered data, suggesting that highly unreliable or incoherent raw angular measurements are already discarded by the system.

\section{B. Assessment of Tracking Methods}

After having presented the system configuration and detailed the fitting parameters, in this section we evaluate the performances of the proposed technique against the ones obtained by two PF-based tracking techniques. The first one employs the Skew t distribution to account for NLOS effects [36], [37], while the second does not employ any NLOS mitigation countermeasures, i.e., it assumes $\Delta d_{t}^{(\ell)}=0 \mathrm{~m}$ in (3). In this second case, all TDoAs are Gaussian distributed, i.e., $p\left(\rho_{t}^{(\ell, k)} \mid \mathbf{u}_{t}\right)=\mathcal{N}\left(\rho_{t}^{(\ell, k)} ; d_{t}^{(\ell, k)}+\mu_{p f}, \sigma_{p f}^{2}\right)$, with fitted parameters $\mu_{p f}=-0.497 \mathrm{~m}$ and $\sigma_{p f}=1.764 \mathrm{~m}$.

As far as the dynamic evolution of the position is concerned, the same model and statistical parameters presented in Sec. II-A are used for all filters. The consistency of the PF solution has been evaluated numerically for different number of particles $N_{\mathrm{P}}$. We choose $N_{\mathrm{P}}=1000$ since any increase beyond this value does not provide any meaningful improvement on the result. Finally, the same initialization process has been used for all tracking filters so as to evaluate the localization performances under the same conditions. To evaluate the performances of the tracking filters we use the Location Root Mean Square Error (RMSE) computed over $N_{\mathrm{TF}}$ realizations as $\operatorname{RMSE}_{t}=\left(\sum_{n=1}^{N_{\mathrm{TF}}} \varepsilon_{t}^{2} / N_{\mathrm{TF}}\right)^{1 / 2}$.

Fig. 15 shows the tracking capabilities of a PF without NLOS compensation (Fig. 15a), of a PF with Skew $t$ modeling of TDoA measurements (Fig. 15b), and of the proposed technique, for both the implementations with only TDoA measurements (Fig. 15c) and with TDoA+AoA (Fig. 15d). The true trajectory is reported as a black solid line, while the estimated locations of each tag are shown as colored dots. 


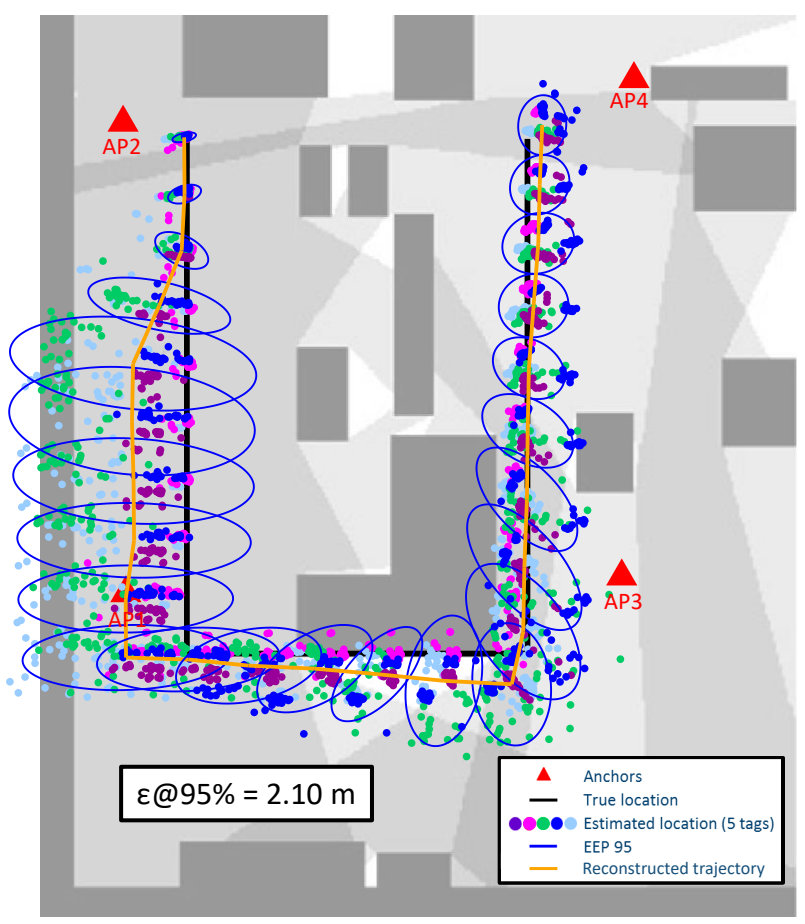

(a) PF without NLOS compensation

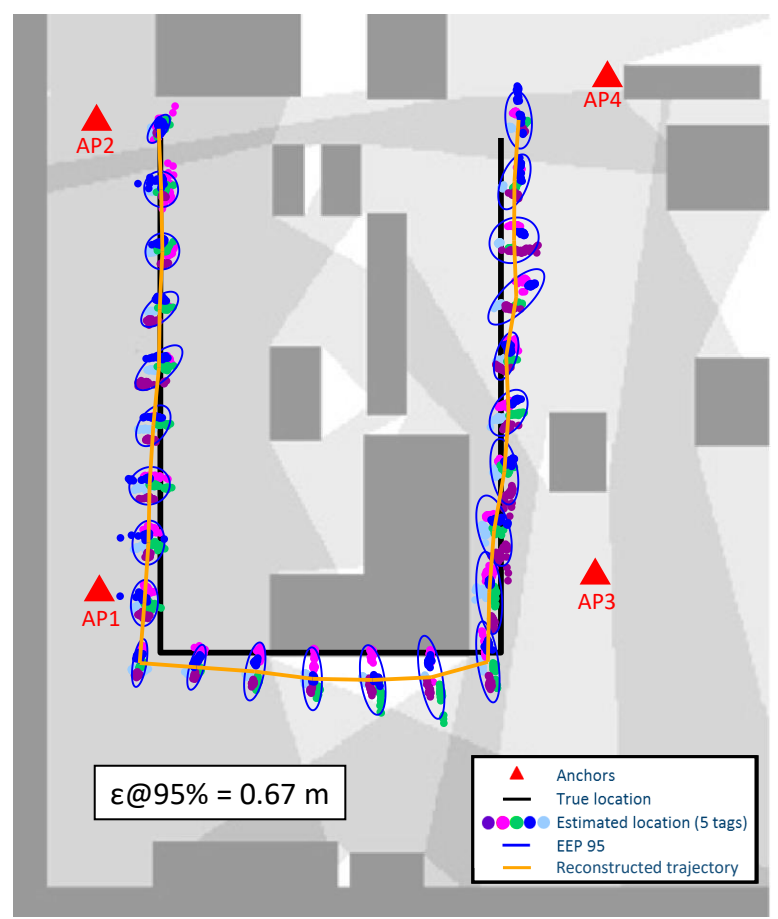

(c) Proposed PF with NLOS compensation (TDoA)

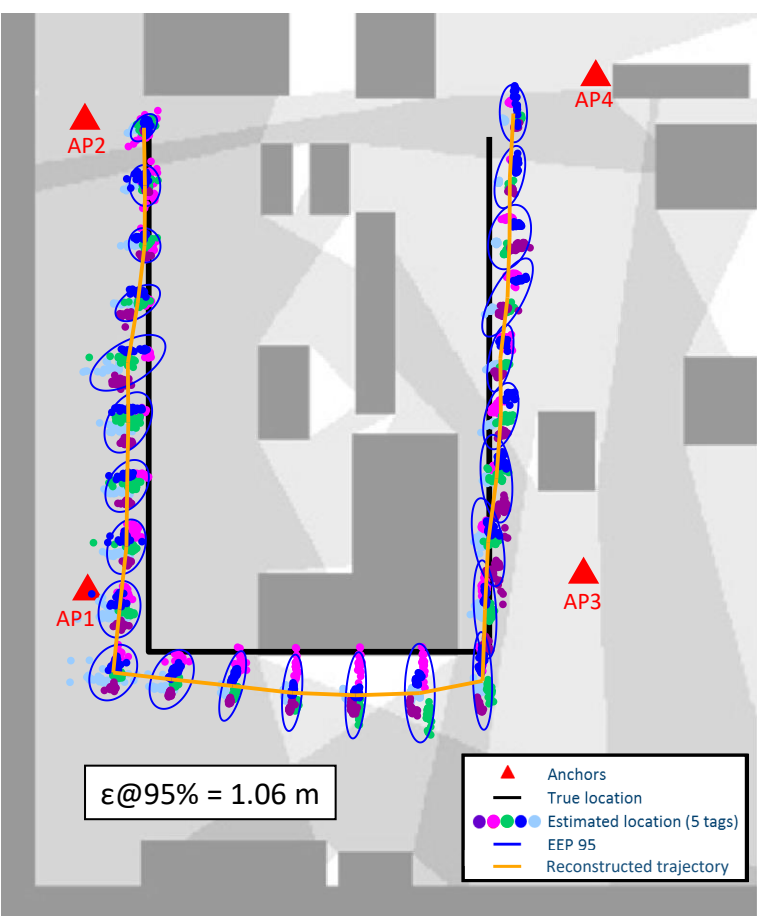

(b) PF with Skew $\mathrm{t}$

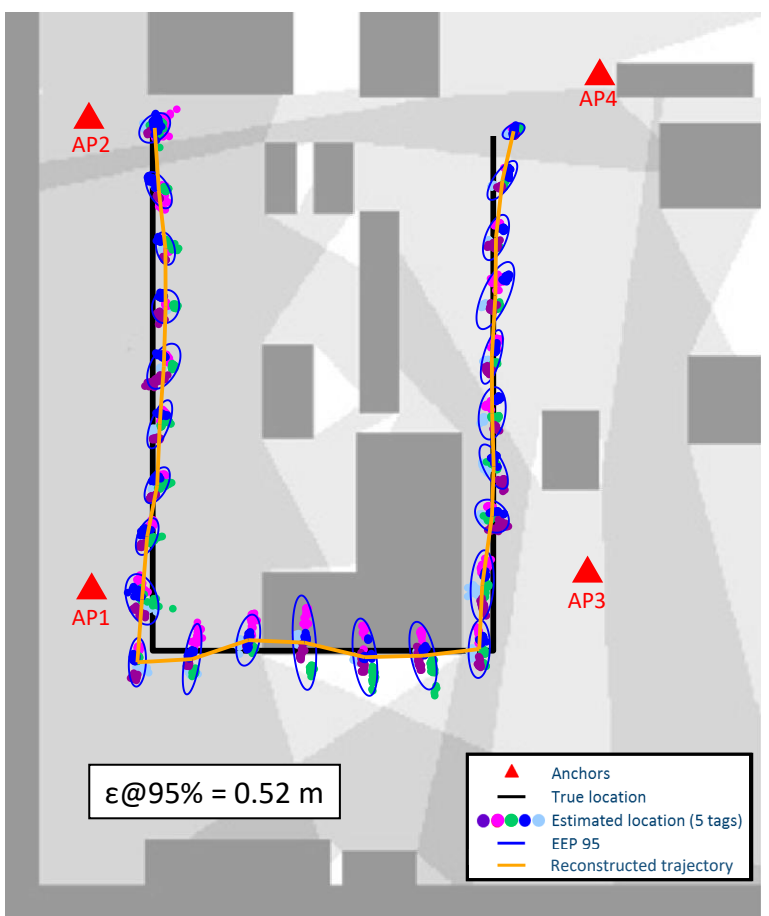

(d) Proposed PF with NLOS compensation (TDoA+AoA)

Fig. 15. Performance comparison of tracking filters localization in the industrial factory: (a) PF without NLOS compensation (b) PF with Skew $t$ (c) Proposed PF with NLOS compensation (TDoA) (d) Proposed PF with NLOS compensation (TDoA+AoA). 


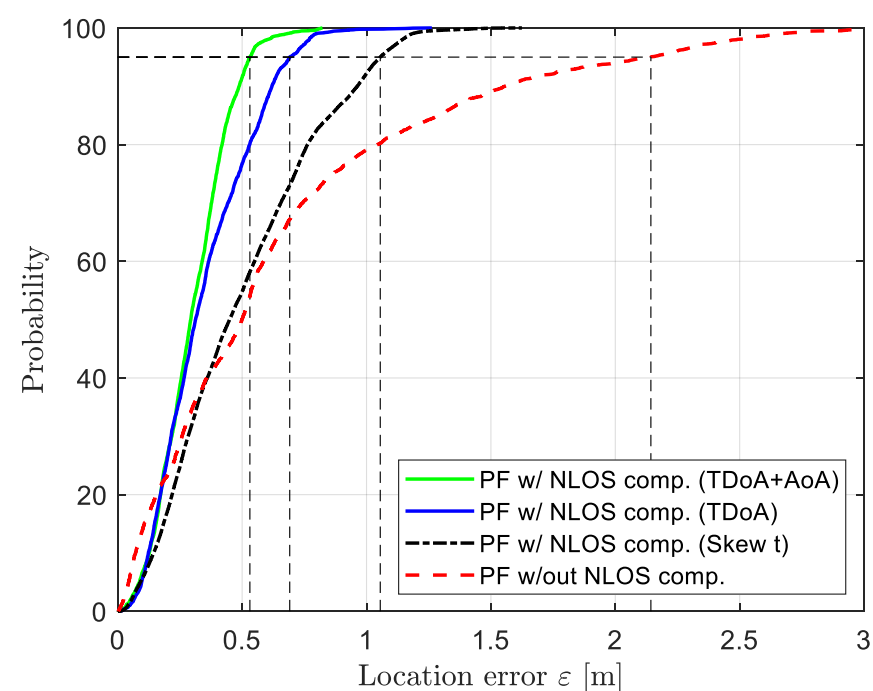

Fig. 16. CDF of the location error of the tracking filters in the industrial environment.

The EEP95 is superposed as the black solid line. The averaged mean over all tags are connected one another with a orange solid line to highlight the reconstructed trajectory.

Analyzing the outcomes of Fig. 15 we highlight that the proposed NLOS compensation with and without AoA information outperforms the other two baseline approaches of Fig. 15a and Fig. 15b. In particular, the reconstructed trajectory (orange line) of Fig. $15 \mathrm{c}$ and $15 \mathrm{~d}$ is closer to the true path (black line). It can be also noticed that the AoA information is particularly helpful in the bottom part of the trajectory (between AP1 and AP3). On the other hand, the orange lines of the Skew tbased filter and PF without NLOS compensation are subject to high positions biases resulting in a mismatch between true and estimated trajectories.

Comparing with the legacy Ubisense performance in Fig. 9a, the location error of the tracking methods in Fig. 15c and in Fig. 15d are higher. However, it is important to recall that Ubisense uses both TDoA and all AoA measurements and can access built-in motion detector to discriminate stationary/mobility conditions, thus adapting the localization algorithm. Conversely, the proposed work aims to demonstrate the advantage of embedding prior information on the scenario of propagation (i.e., LOS/NLOS conditions) into the filtering approach by the JMS framework. The proposed approach has been also shown to provide higher positioning accuracy when the azimuth AoA information is properly integrated within the localization framework. In addition, it could be possible to exploit also the elevation information to refine the sight state detection and provide even more precise results. This further integration is not considered here as this would require a 3D floorplan of the considered scenario to fully characterize the sight conditions along the vertical dimension. It is not trivial nor common to get such additional information as it requires a $3 \mathrm{D}$ mapping of the environment, which also needs to be constantly updated any time new furniture or assets are moved. It comes that it is hardly achievable and feasible in an industrial site with operation activities.

The overall performance gain of the proposed augmentation

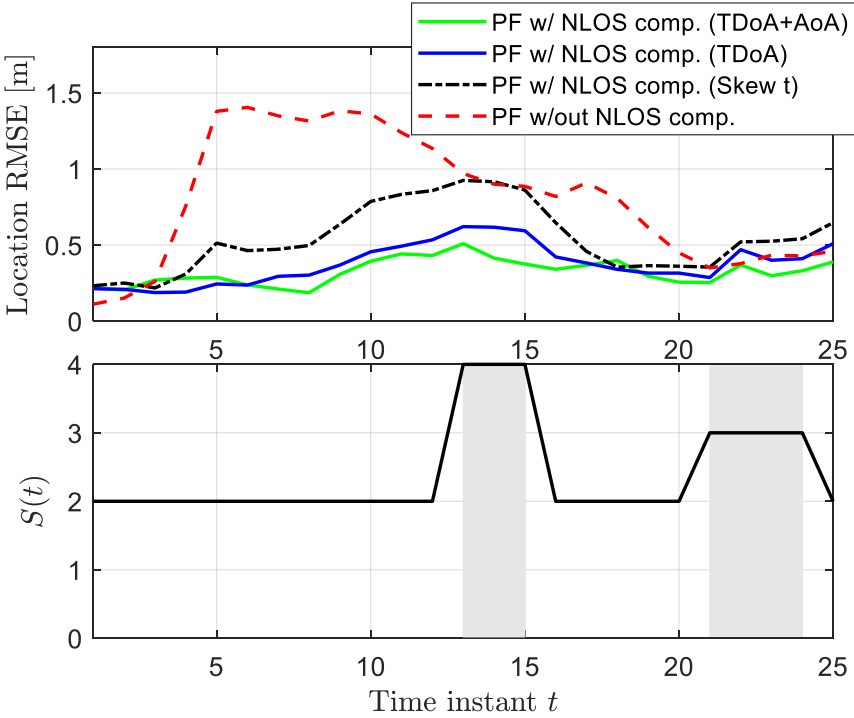

Fig. 17. Tracking filters localization results: comparison among PF filters in terms of location RMSE (top) and sum of sight conditions values for each location (bottom).

technique with and without the azimuth angles can be appreciated by looking at Fig. 16, where the CDF of location error is reported by aggregating the outcomes provided by all tags for all positions. The PF with NLOS compensation that exploits TDoA and AoA achieves a localization error $\varepsilon$ less than 53 $\mathrm{cm}$ in $95 \%$ of the cases, whereas the compensation approach that relies only on TDoA measurements attains a slightly larger value of $67 \mathrm{~cm}$. On the other hand, the two baseline approaches exhibit a much larger error: the PF with the Skew t obtains a positioning error less than $1.06 \mathrm{~m}$ in $95 \%$ of the cases, while the PF without NLOS compensation a large $2.10 \mathrm{~m}$. This confirms that the proposed approach is able to reduce false localization due to NLOS propagation, particularly if the AoA information is combined with the TDoA.

The upper part of Fig. 17 shows the location RMSE and the aggregated sight condition values $S(t)=\sum_{\ell=1}^{L} \mathbf{s}_{t}^{(\ell)}$ for each time step (or, equivalently, tag position). Comparing the results, it can be seen that the the PF without NLOS compensation performs rather poorly for $4 \leq t \leq 20$, where the location RMSE reaches values up to $1.4 \mathrm{~m}$. This is expected since most of the location considered are heavily subjected to NLOS conditions as confirmed by Fig. 11a where large TDoA ranging errors are reported. The PF with Skew $t$ better performs compared to the PF without NLOS compensation but it is not able to completely compensate NLOS propagation from $5 \leq t \leq 15$. In fact, from $t=5$ onward many TDoAs are affected by large errors that are outside the support of the Skew $\mathrm{t}$ distribution and cannot be lowered. As the time $t$ increases, the location errors accumulates and the PF with Skew $t$ attains almost the same performance of the PF without NLOS propagation for $13 \leq t \leq 15$. On the other hand, the TDoA and TDoA+AoA filters are able to compensate the NLOS effects since the location RMSE for $4 \leq t \leq 20$ is heavily reduced compared to the other two methods analyzed before. In these positions, the integration of the AoA information yields better results compared to the TDoA-only approach, 


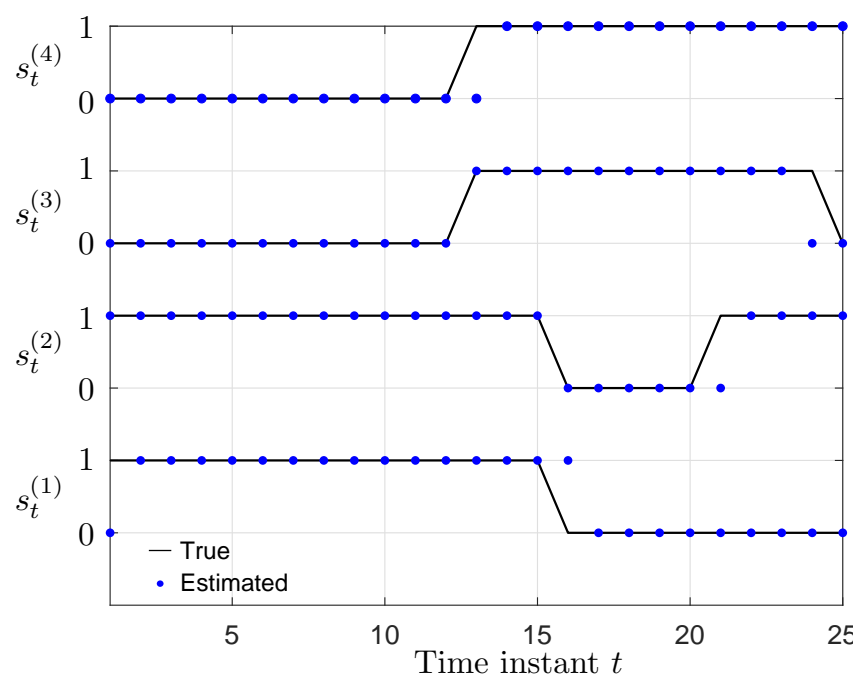

Fig. 18. Comparison between estimated sight conditions (LOS/NLOS) and real wireless propagating conditions extracted from coverage maps.

making the overall solution more robust to NLOS propagation. Higher accuracy is reached in the area between AP1 and AP2 rather than between AP3 and AP4, as expected being the NLOS contribution more pronounced in the vicinity of AP1/AP2 compared to the neighborhood of AP3/AP4. In fact, the overall TDoA distribution of Fig. 11a for $5 \leq t \leq 10$ shows high error components while for $15 \leq t \leq 20$ larger errors are detected more sparingly.

The estimated sight conditions $s_{t}^{(\ell)}$ of all APs (black dots) are compared to the ones extracted from the LOS/NLOS coverage maps in Fig. 18 (black solid lines). Analyzing the results, only few location points present an incorrect sight condition (in correspondence of the variation) while the majority of them exhibit correct LOS/NLOS values. These results confirm that the use of first-order Markov modeling for the sight conditions is effective and no advanced (but heavier from a computational point of view) techniques are demanded. Moreover, the localization precision is not that much affected by sight estimation errors, thus the proposed method is robust against non-prefect LOS/NLOS state information.

\section{Assessment of TDoA Ranging Error Compensation}

To fully characterize the performances of the proposed approach for NLOS compensation, as a final analysis we provide a comparison on the estimated TDoA ranges obtained by the proposed TDoA-only PF with NLOS mitigation with respect to the raw TDoA provided by the Ubisense system. This comparison is reported in Fig. 19, where the estimated TDoA (averaged over the available measurements for each position of the trajectory) is plotted over time. The comparison considers the proposed filter with NLOS compensation in the version of TDoA-only availability (black solid curve), the average TDoA provided by the Ubisense system output (red solid curve) and the true TDoA (black dashed line). Besides the average values, we also indicate with the shaded area the confidence of the estimate associated to the standard deviation. To ease the visualization of the results, we manipulated all TDoAs such that the master anchor is always the first anchor (i.e., AP1).
This allows to visualize all results considering the anchor pairs AP1-AP2, AP1-AP3, and AP1-AP4. Comparing the results, the proposed TDoA-only compensation approach better copes with the NLOS degradation rather than the Ubisense outcomes. In fact, for most of the positions of the trajectory, the reconstructed TDoAs of the proposed methods are always close to the true value, while the TDoAs estimated by Ubisense suffers from a severe NLOS degradation. This analysis confirms that the proposed approach is able to compensate for the large errors caused by severe multipath and NLOS propagation and improve the the estimation precision of TDoA of commercial solutions.

\section{CONCLUSIONS}

This work addressed the problem of UWB localization in IIoT applications. A novel Bayesian augmentation technique has been proposed for NLOS mitigation and validated in a real industrial environment. An extensive measurement campaign has been carried out, using commercial UWB devices. After a first assessment of different technologies (Decawave, Sewio and Ubisense) in both laboratory and industrial facilities, the Ubisense system was selected for an in-depth analysis of NLOS effects in harsh indoor propagation conditions and for the assessment of the proposed augmentation methods as it allows the extraction of information related to the physicallayer. Raw TDoA and AoA UWB data were extracted from the industrial campaign and used for validating the developed filter. Experimental results show that it is possible to reduce the positioning error from approximately $2.10 \mathrm{~m}$ down to $67 \mathrm{~cm}$ (on average) in a very complex multipath environment inside a real factory using only 4 APs overall, with an average $60 \%$ of time in NLOS condition exploiting only TDoA measurements. If the AoA are also integrated in the proposed method, the localization error can be further reduced to $52 \mathrm{~cm}$. To better characterize the performances of the developed approach with respect to other works, a comparison with a state-of-the-art technique is presented. Results show that the compensation approach proposed herein is able to outperform also the stateof-the-art method, which achieves a positioning error of 1.06 m compared to $67 \mathrm{~cm}$ obtained by the TDoA-only PF with NLOS compensation.

Thus, the developed NLOS mitigation technique is effective in reducing false localization in harsh industrial environments and it is able to deliver highly accurate responses that are appropriate for most IIoT applications to guarantee reliable and robust operation. Furthermore, the proposed augmentation system is robust against non-perfect estimation of the sight conditions experienced at the reference stations. Further research activity will target a possible development of a mitigation system capable of learning the NLOS statistics, starting from a rough information of the sight conditions and updating the map while tracking the target.

\section{APPENDIX A}

In this Appendix, we explain in detail the PF methodology used in the paper. The estimate of the composite state $\mathbf{x}_{t}$ can be obtained at any time instant $t$ through the recursive 


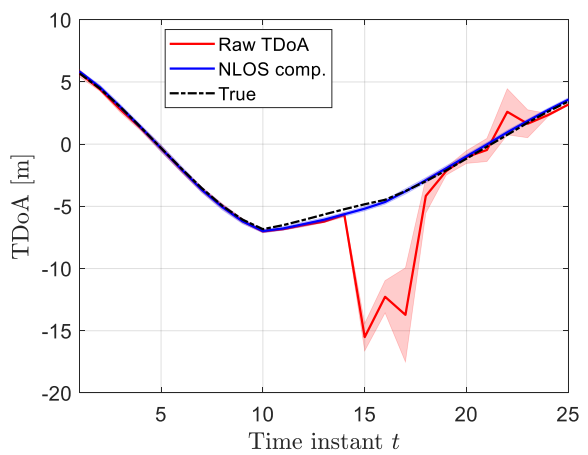

(a) TDoA $1-2$

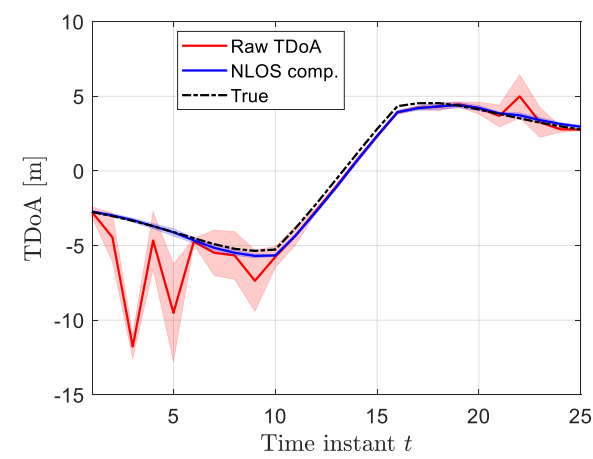

(b) TDoA 1-3

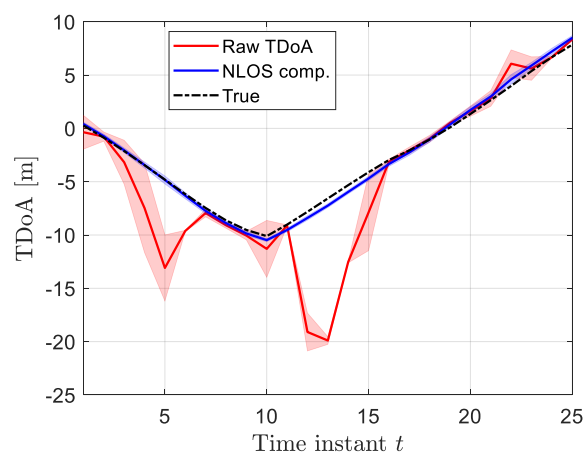

(c) TDoA 1-4

Fig. 19. TDoA ranging estimates analysis: black solid line indicates the true TDoA over the whole trajectory points, black solid line the estimated TDoA from the NLOS compensation approach (TDoA-only) and red solid line the raw TDoA estimated by Ubisense.

computation of (7) and (8). At first, the a-priori pdf of equation (8) can be approximated as the sum of $N_{\mathrm{P}}$ independent and identically distributed (i.i.d.) Dirac's pulses $\left\{\mathbf{u}_{t}^{(n)}, \mathbf{s}_{t}^{(n)}\right\}_{n=1}^{N_{\mathrm{P}}}$ equally weighted as:

$$
p\left(\mathbf{u}_{t}, \mathbf{s}_{t} \mid \boldsymbol{\Omega}_{1: t-1}\right) \approx \frac{1}{N_{\mathrm{P}}} \sum_{n=1}^{N_{\mathrm{P}}} \delta\left(\mathbf{u}_{t}-\mathbf{u}_{t}^{(n)}\right) \delta\left[\mathbf{s}_{t}-\mathbf{s}_{t}^{(n)}\right],
$$

where the particle subset $\mathbf{u}_{t}^{(n)}$ can assume any continuous value within the $2 \mathrm{D}$ space, while $\mathbf{s}_{t}^{(n)}$ is defined over the discrete set $\mathcal{S}$. Using (7) and (19) the a posteriori probability is approximated as:

$$
\begin{aligned}
p\left(\mathbf{x}_{t} \mid \boldsymbol{\Omega}_{1: t}\right) & \approx \frac{1}{N_{\mathrm{P}}} \sum_{n=1}^{N_{\mathrm{P}}} p\left(\boldsymbol{\Omega}_{t} \mid \mathbf{u}_{t}, \mathbf{s}_{t}\right) \delta\left(\mathbf{u}_{t}-\mathbf{u}_{t}^{(n)}\right) \delta\left[\mathbf{s}_{t}-\mathbf{s}_{t}^{(n)}\right] \\
& =\sum_{n=1}^{N_{\mathrm{P}}} \widetilde{w}_{t}^{(n)} \delta\left(\mathbf{u}_{t}-\mathbf{u}_{t}^{(n)}\right) \delta\left[\mathbf{s}_{t}-\mathbf{s}_{t}^{(n)}\right],
\end{aligned}
$$

where $\widetilde{w}_{t}^{(n)}=p\left(\boldsymbol{\Omega}_{t} \mid \mathbf{u}_{t}^{(n)}, \mathbf{s}_{t}^{(n)}\right) / N_{\mathrm{P}}$. After normalization, (20) reduces to:

$$
p\left(\mathbf{u}_{t}, \mathbf{s}_{t} \mid \boldsymbol{\Omega}_{1: t}\right)=\sum_{n=1}^{N_{\mathrm{P}}} w_{t}^{(n)} \delta\left(\mathbf{u}_{t}-\mathbf{u}_{t}^{(n)}\right) \delta\left[\mathbf{s}_{t}-\mathbf{s}_{t}^{(n)}\right],
$$

where $w_{t}^{(n)}=\widetilde{w}_{t}^{(n)} / \sum_{n=1}^{N_{\mathrm{P}}} \widetilde{w}_{t}^{(n)}$. Then, the MMSE position estimated can be obtained as $\widehat{\mathbf{u}}_{t}^{(n)}=\sum_{n=1}^{N_{\mathrm{P}}} w_{t}^{(n)} \mathbf{u}_{t}^{(n)}$, while the estimated sight condition is $\widehat{\mathbf{s}}_{t}^{(n)}=\operatorname{dec}\left(\sum_{n=1}^{N_{\mathrm{P}}} w_{t}^{(n)} \mathbf{s}_{t}^{(n)}\right)$, where $\operatorname{dec}(x)$ is a function that is equal to 1 when $x \geq 1 / 2$ and 0 otherwise.

The last step required when dealing with $\mathrm{PF}$ is the resampling strategy. In fact, in order to model the evolution of each particle from the joint state $\left(\mathbf{u}_{t}^{(n)}, \mathbf{s}_{t}^{(n)}\right)$ to the next one $\left(\mathbf{u}_{t+1}^{(n)}, \mathbf{s}_{t+1}^{(n)}\right)$ and evaluate $p\left(\mathbf{x}_{t+1} \mid \boldsymbol{\Omega}_{1: t+1}\right)$, it is required to compute the sequential importance resampling [62]. The current set of particles $\left\{\mathbf{u}_{t}^{(n)}, \mathbf{s}_{t}^{(n)}\right\}_{n=1}^{N_{\mathrm{P}}}$ is resampled, either deterministically or randomly [62], so as to obtain a new set $\left\{\widetilde{\mathbf{u}}_{t}^{(n)}, \widetilde{\mathbf{s}}_{t}^{(n)}\right\}$ such that $P\left(\widetilde{\mathbf{u}}_{t}^{(n)}=\mathbf{u}_{t}^{(n)}, \widetilde{\mathbf{s}}_{t}^{(n)}=\mathbf{s}_{t}^{(n)}\right)=$ $w_{t}^{(n)}, \forall n$. After this step, particles with negligible weights are discarded in favor of stronger weights which are reallocated over a new set with equal values $\widetilde{w}_{t}^{(n)}=N_{\mathrm{P}}^{-1}$.

\section{REFERENCES}

[1] S. Gezici, Zhi Tian, G. B. Giannakis, H. Kobayashi, A. F. Molisch, H. V. Poor, and Z. Sahinoglu, "Localization via ultra-wideband radios: a look at positioning aspects for future sensor networks," IEEE Signal Processing Magazine, vol. 22, no. 4, pp. 70-84, Jul. 2005.

[2] M. Z. Win and R. A. Scholtz, "Characterization of ultra-wide bandwidth wireless indoor channels: a communication-theoretic view," IEEE Journal on Selected Areas in Communications, vol. 20, no. 9, pp. 1613-1627, Dec. 2002.

[3] — , "Impulse radio: how it works," IEEE Communications Letters, vol. 2, no. 2, pp. 36-38, Feb. 1998.

[4] Y. Gu, A. Lo, and I. Niemegeers, "A survey of indoor positioning systems for wireless personal networks," IEEE Communications Surveys Tutorials, vol. 11, no. 1, pp. 13-32, Mar. 2009.

[5] A. Alarifi, A. Al-Salman, M. Alsaleh, A. Alnafessah, S. Alhadhrami, M. Al-Ammar, and H. Al-Khalifa, "Ultra wideband indoor positioning technologies: Analysis and recent advances," Sensors, vol. 16, pp. 1-36, May 2016.

[6] M. Z. Win and R. A. Scholtz, "On the energy capture of ultrawide bandwidth signals in dense multipath environments," IEEE Communications Letters, vol. 2, no. 9, pp. 245-247, Sep. 1998.

[7] — , "On the robustness of ultra-wide bandwidth signals in dense multipath environments," IEEE Communications Letters, vol. 2, no. 2, pp. 51-53, Feb. 1998

[8] Apple, "Apple - iPhone 11," https://www.apple.com/iphone-11.

[9] J. Wang, A. K. Raja, and Z. Pang, "Prototyping and experimental comparison of IR-UWB based high precision localization technologies," in 2015 IEEE 12th Intl Conf on Ubiquitous Intelligence and Computing and 2015 IEEE 12th Intl Conf on Autonomic and Trusted Computing and 2015 IEEE 15th Intl Conf on Scalable Computing and Communications and Its Associated Workshops (UIC-ATC-ScalCom), Aug. 2015, pp. $1187-1192$.

[10] A. Albaidhani, A. Morell, and J. L. Vicario, "Ranging in UWB using commercial radio modules: Experimental validation and NLOS mitigation," in 2016 International Conference on Indoor Positioning and Indoor Navigation, Oct. 2016, pp. 1-7.

[11] A. R. Jimnez and F. Seco, "Comparing Decawave and Bespoon UWB location systems: indoor/outdoor performance analysis," in 2016 International Conference on Indoor Positioning and Indoor Navigation, Oct. 2016, pp. 1-8.

[12] A. R. Jimnez Ruiz and F. Seco Granja, "Comparing Ubisense, BeSpoon, and DecaWave UWB location systems: Indoor performance analysis," IEEE Transactions on Instrumentation and Measurement, vol. 66, no. 8, pp. 2106-2117, Aug. 2017.

[13] B. Silva and G. P. Hancke, "IR-UWB-based non-line-of-sight identification in harsh environments: Principles and challenges," IEEE Transactions on Industrial Informatics, vol. 12, no. 3, pp. 1188-1195, Jun. 2016.

[14] R. Simedroni, E. Puschita, T. Palade, P. Dolea, C. Codau, R. Buta, and A. Pastrav, "Indoor positioning using Decawave MDEK1001," in 2020 International Workshop on Antenna Technology, May 2020, pp. 1-4.

[15] E. Puschita, R. Simedroni, T. Palade, C. Codau, S. Vos, V. Ratiu, and O. Ratiu, "Performance evaluation of the UWB-based CDS indoor 
positioning solution," in 2020 International Workshop on Antenna Technology (iWAT), Feb. 2020, pp. 1-4.

[16] S. He and X. Dong, "High-accuracy localization platform using asynchronous time difference of arrival technology," IEEE Transactions on Instrumentation and Measurement, vol. 66, no. 7, pp. 1728-1742, Jul. 2017.

[17] Y. Zhou, C. L. Law, Y. L. Guan, and F. Chin, "Indoor elliptical localization based on asynchronous UWB range measurement," IEEE Transactions on Instrumentation and Measurement, vol. 60, no. 1, pp. 248-257, Jan. 2011.

[18] A. Cazzorla, G. De Angelis, A. Moschitta, M. Dionigi, F. Alimenti, and P. Carbone, "A 5.6-GHz UWB position measurement system," IEEE Transactions on Instrumentation and Measurement, vol. 62, no. 3, pp. 675-683, Mar. 2013.

[19] P. Krape and M. Munih, "Anchor calibration for real-time-measurement localization systems," IEEE Transactions on Instrumentation and Measurement, vol. 69, no. 12, pp. 9907-9917, Dec. 2020.

[20] J. Sidorenko, V. Schatz, N. Scherer-Negenborn, M. Arens, and U. Hugentobler, "Error corrections for ultrawideband ranging," IEEE Transactions on Instrumentation and Measurement, vol. 69, no. 11, pp. 9037-9047, Nov. 2020.

[21] K. Zhao, M. Zhu, B. Xiao, X. Yang, C. Gong, and J. Wu, "Joint RFID and UWB technologies in intelligent warehousing management system," IEEE Internet of Things Journal, pp. 1-1, May 2020.

[22] D. Dardari, A. Conti, U. Ferner, A. Giorgetti, and M. Z. Win, "Ranging with ultrawide bandwidth signals in multipath environments," Proceedings of the IEEE, vol. 97, no. 2, pp. 404-426, Feb. 2009.

[23] J. Khodjaev, Y. Park, and A. Saeed Malik, "Survey of NLOS identification and error mitigation problems in UWB-based positioning algorithms for dense environments," Annals of telecommunications annales des télécommunications, vol. 65, no. 5, pp. 301-311, Jun. 2010. [Online]. Available: https://doi.org/10.1007/s12243-009-0124-Z

[24] J. Borras, P. Hatrack, and N. B. Mandayam, "Decision theoretic framework for NLOS identification," in VTC '98. 48th IEEE Vehicular Technology Conference. Pathway to Global Wireless Revolution, vol. 2, May 1998, pp. $1583-1587$.

[25] İ. Güvenç, C.-C. Chong, and F. Watanabe, "NLOS identification and mitigation for UWB localization systems," in 2007 IEEE Wireless Communications and Networking Conference, Mar. 2007, pp. 15711576.

[26] İ. Güvenç, C.-C. Chong, F. Watanabe, and H. Inamura, "NLOS identification and weighted least-squares localization for UWB systems using multipath channel statistics," EURASIP Journal on Advances in Signal Processing, vol. 2008, pp. 1-14, Jan. 2007.

[27] C. Morelli, M. Nicoli, V. Rampa, and U. Spagnolini, "Hidden Markov models for radio localization in mixed LOS/NLOS conditions," IEEE Transactions on Signal Processing, vol. 55, no. 4, pp. 1525-1542, Apr. 2007.

[28] M. Nicoli, C. Morelli, and V. Rampa, "A jump Markov particle filter for localization of moving terminals in multipath indoor scenarios," IEEE Transactions on Signal Processing, vol. 56, no. 8, pp. 3801-3809, Aug. 2008.

[29] B. L. Le, K. Ahmed, and H. Tsuji, "Mobile location estimator with NLOS mitigation using Kalman filtering," in 2003 IEEE Wireless Communications and Networking, vol. 3, Mar. 2003, pp. 1969-1973.

[30] B. Cao, S. Wang, S. Ge, X. Ma, and W. Liu, "A novel mobile target localization approach for complicate underground environment in mixed LOS/NLOS scenarios," IEEE Access, vol. 8, pp. 96347-96362, May 2020.

[31] W. Suski, S. Banerjee, and A. Hoover, "Using a map of measurement noise to improve UWB indoor position tracking," IEEE Transactions on Instrumentation and Measurement, vol. 62, no. 8, pp. 2228-2236, Aug. 2013.

[32] X. Zhu, J. Yi, J. Cheng, and L. He, "Adapted error map based mobile robot UWB indoor positioning," IEEE Transactions on Instrumentation and Measurement, vol. 69, no. 9, pp. 6336-6350, Sep. 2020.

[33] Q. Tian, K. I. Wang, and Z. Salcic, "A resetting approach for INS and UWB sensor fusion using particle filter for pedestrian tracking," IEEE Transactions on Instrumentation and Measurement, vol. 69, no. 8, pp. 5914-5921, Aug. 2020.

[34] J. D. Hol, F. Dijkstra, H. Luinge, and T. B. Schon, "Tightly coupled UWB/IMU pose estimation," in 2009 IEEE International Conference on Ultra-Wideband, Oct. 2009, pp. 688-692.

[35] D. Feng, C. Wang, C. He, Y. Zhuang, and X. Xia, "Kalman-filter-based integration of IMU and UWB for high-accuracy indoor positioning and navigation," IEEE Internet of Things Journal, vol. 7, no. 4, pp. 31333146, Jan. 2020.
[36] H. Nurminen, T. Ardeshiri, R. Pich, and F. Gustafsson, "A NLOS-robust TOA positioning filter based on a skew-t measurement noise model," in 2015 International Conference on Indoor Positioning and Indoor Navigation (IPIN), Dec. 2015, pp. 1-7.

[37] — - "Robust inference for state-space models with skewed measurement noise," IEEE Signal Processing Letters, vol. 22, no. 11, pp. 18981902, May 2015.

[38] Y. Huang, Y. Zhang, P. Shi, Z. Wu, J. Qian, and J. A. Chambers, "Robust Kalman filters based on Gaussian scale mixture distributions with application to target tracking," IEEE Transactions on Systems, Man, and Cybernetics: Systems, vol. 49, no. 10, pp. 2082-2096, Dec. 2019.

[39] Y. Huang, Y. Zhang, Y. Zhao, L. Mihaylova, and J. A. Chambers, "Robust RauchTungStriebel smoothing framework for heavy-tailed and/or skew noises," IEEE Transactions on Aerospace and Electronic Systems, vol. 56, no. 1, pp. 415-441, May 2020.

[40] B. Cao, S. Wang, S. Ge, and W. Liu, "Improving positioning accuracy of UWB in complicated underground NLOS scenario using calibration, VBUKF, and WCA," IEEE Transactions on Instrumentation and Measurement, vol. 70, pp. 1-13, 2021.

[41] S. Maran, W. M. Gifford, H. Wymeersch, and M. Z. Win, "NLOS identification and mitigation for localization based on UWB experimental data," IEEE Journal on Selected Areas in Communications, vol. 28 , no. 7, pp. 1026-1035, Sep. 2010.

[42] T. Van Nguyen, Y. Jeong, H. Shin, and M. Z. Win, "Machine learning for wideband localization," IEEE Journal on Selected Areas in Coтmunications, vol. 33, no. 7, pp. 1357-1380, Jul. 2015.

[43] H. Wymeersch, G. Seco-Granados, G. Destino, D. Dardari, and F. Tufvesson, " $5 \mathrm{G}$ mmwave positioning for vehicular networks," IEEE Wireless Communications, vol. 24, no. 6, pp. 80-86, Dec. 2017.

[44] COST Action CA15104, IRACON, T. Pedersen, and B. Fleury, "Whitepaper on new localization methods for 5G wireless systems and the internet-of-things," 2018.

[45] C. Gentner, T. Jost, W. Wang, S. Zhang, A. Dammann, and U. Fiebig, "Multipath assisted positioning with simultaneous localization and mapping," IEEE Transactions on Wireless Communications, vol. 15, no. 9, pp. 6104-6117, Sep. 2016.

[46] E. Leitinger, P. Meissner, M. Lafer, and K. Witrisal, "Simultaneous localization and mapping using multipath channel information," in 2015 IEEE International Conference on Communication Workshop, Jun. 2015, pp. $754-760$.

[47] R. Mendrzik, F. Meyer, G. Bauch, and M. Z. Win, "Enabling situational awareness in millimeter wave massive MIMO systems," IEEE Journal of Selected Topics in Signal Processing, vol. 13, no. 5, pp. 1196-1211, Sep. 2019.

[48] R. Mendrzik, H. Wymeersch, G. Bauch, and Z. Abu-Shaban, "Harnessing NLOS components for position and orientation estimation in 5G millimeter wave MIMO," IEEE Transactions on Wireless Communications, vol. 18, no. 1, pp. 93-107, Jan. 2019.

[49] Decawave, "About us - Decawave," https://www.decawave.com/ about-us/.

[50] Ubisense, "Ubisense: Enterprise Location Intelligence Solutions," https: //www.ubisense.net/.

[51] Sewio, "Real-time location systems (RTLS) for indoor tracking," https: //www.sewio.net/.

[52] L. Barbieri, M. Brambilla, R. Pitic, A. Trabattoni, S. Mervic, and M. Nicoli, "UWB real-time location systems for smart factory: Augmentation methods and experiments," in 2020 IEEE 31st Annual International Symposium on Personal, Indoor and Mobile Radio Communications, Aug. 2020, pp. 1-7.

[53] F. Gustafsson and F. Gunnarsson, "Mobile positioning using wireless networks: possibilities and fundamental limitations based on available wireless network measurements," IEEE Signal Processing Magazine, vol. 22, no. 4, pp. 41-53, Jun. 2005.

[54] A. F. Molisch, "Ultra-Wide-Band propagation channels," Proceedings of the IEEE, vol. 97, no. 2, pp. 353-371, Mar. 2009.

[55] Z. Irahhauten, H. Nikookar, and G. J. M. Janssen, "An overview of ultra wide band indoor channel measurements and modeling," IEEE Microwave and Wireless Components Letters, vol. 14, no. 8, pp. 386388, Jul. 2004.

[56] R. J. . Cramer, R. A. Scholtz, and M. Z. Win, "Evaluation of an ultrawide-band propagation channel," IEEE Transactions on Antennas and Propagation, vol. 50, no. 5, pp. 561-570, May 2002.

[57] A. Molisch, K. Balakrishnan, D. Cassioli, C.-C. Chong, S. Emami, A. Fort, J. Karedal, J. Kunisch, H. Schantz, U. Schuster, and K. Siwiak, “IEEE 802.15.4a Channel Model - Final Report," IEEE P802, Tech. Rep., 2004. 
[58] S. Kotz, T. Kozubowski, and K. Podgorski, The Laplace Distribution and Generalizations. Springer Science \& Business Media, Jan. 2001.

[59] Q. H. Spencer, B. D. Jeffs, M. A. Jensen, and A. L. Swindlehurst, "Modeling the statistical time and angle of arrival characteristics of an indoor multipath channel," IEEE Journal on Selected Areas in Communications, vol. 18, no. 3, pp. 347-360, Mar. 2000.

[60] R. B. Ertel, P. Cardieri, K. W. Sowerby, T. S. Rappaport, and J. H. Reed, "Overview of spatial channel models for antenna array communication systems," IEEE Personal Communications, vol. 5, no. 1, pp. 10-22, Feb. 1998.

[61] Y. Zhang, A. K. Brown, W. Q. Malik, and D. J. Edwards, "High resolution 3-D angle of arrival determination for indoor UWB multipath propagation," IEEE Transactions on Wireless Communications, vol. 7 , no. 8, pp. 3047-3055, Aug. 2008.

[62] M. S. Arulampalam, S. Maskell, N. Gordon, and T. Clapp, "A tutorial on particle filters for online nonlinear/non-Gaussian Bayesian tracking," IEEE Transactions on Signal Processing, vol. 50, no. 2, pp. 174-188, Feb. 2002.

[63] Y. Kalambet, Y. Kozmin, K. Mikhailova, I. Nagaev, and P. Tikhonov, "Reconstruction of chromatographic peaks using the exponentially modified Gaussian function," Journal of Chemometrics, vol. 25, no. 7, pp. 352-356, Feb. 2011.

[64] W. Reed, The normal-Laplace distribution and its relatives. Springer, Jan. 2006, pp. 61-74.

[65] I. S. Association et al., "IEEE Standard for Local and metropolitan area networks-Part 15.4: Low-Rate Wireless Personal Area Networks (LRWPANs)," IEEE Std 802.15.4-2011 (Revision of IEEE Std 802.15.42006), pp. 1-314, Sep. 2011.

[66] A. F. Molisch, "Ultrawideband propagation channels-theory, measurement, and modeling," IEEE Transactions on Vehicular Technology, vol. 54, no. 5, pp. 1528-1545, Nov. 2005.

[67] A. F. Molisch, D. Cassioli, C. Chong, S. Emami, A. Fort, B. Kannan, J. Karedal, J. Kunisch, H. G. Schantz, K. Siwiak, and M. Z. Win, "A comprehensive standardized model for ultrawideband propagation channels," IEEE Transactions on Antennas and Propagation, vol. 54 no. 11, pp. 3151-3166, Nov. 2006.

[68] B. Kannan, K. C. Wee, S. Xu, C. L. Chuan, F. Chin, C. Y. Huat, C. C. Choy, T. T. Thiang, P. Xiaoming, M. Ong and S. Krishnan, "UWB channel characterization in indoor office environments," IEEE, Tech. Rep., 2004.

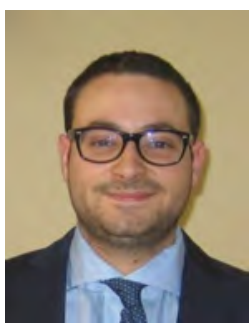

Luca Barbieri received the B.Sc. and M.Sc. (cum laude) degrees in Telecommunication Engineering from Politecnico di Milano in 2017 and 2019, respectively. He is currently a Ph.D. student in Information Technology at Dipartimento di Elettronica, Informazione e Bioingegneria (DEIB), Politecnico di Milano. His current research interests focus on machine learning and localization techniques for vehicular and industrial networks.

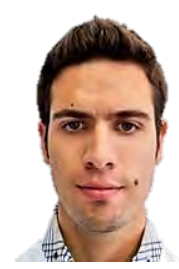

Mattia Brambilla received the B.Sc. and M.Sc. degrees in Telecommunication Engineering and the Ph.D. degree (cum laude) in Information Technology from the Politecnico di Milano, in 2015, 2017, and 2021, respectively. He was a Visiting Researcher with the NATO Centre for Maritime Research and Experimentation (CMRE), La Spezia, Italy, in 2019. $\mathrm{He}$ is currently a Research Fellow with the Politecnico di Milano. His research interests include signal processing, statistical learning, and data fusion for cooperative communication and localization in vehicular, maritime, and industrial environments. He was a recipient of the Best Student Paper Award at the 2018 IEEE Statistical Signal Processing Workshop.

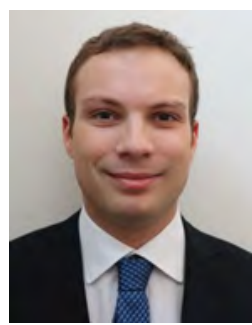

Andrea Trabattoni received the B.Sc. and M.Sc. degrees in Automation and Control engineering from Politecnico di Milano in 2015 and 2017, respectively. From 2017 he worked as Digital Factory engineer in $R \& D$ Process and equipment department at Pirelli Tyre, with focus on Factory IT system improvement and usage of RFID to have a complete track\&trace in the tyre manufacturing process. Since April 2021 Andrea is working in Haier Europe, to improve traceability systems in the household appliances production field.

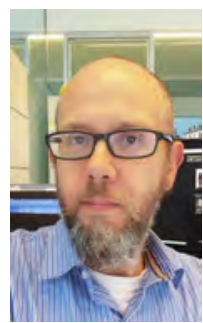

Stefano Mervic received the M.Sc. degree in Information Science at Università degli Studi di Milano in 1996. He is currently Head of P\&E Digital Transformation at Pirelli Tyre.



Monica Nicoli received the M.Sc. degree (cum laude) in Telecommunication Engineering (1998) and the Ph.D. degree in Electronics and Communication Engineering (2002) from Politecnico di Milano. She was a Visiting Researcher with ENI Agip, Italy, from 1998 to 1999 , and also with Uppsala University, Sweden, in 2001. In 2002, she joined Politecnico di Milano as a Faculty Member. She is currently an Associate Professor of Telecommunications with the Department of Management, Economics and Industrial Engineering, Politecnico di Milano. Her research interests cover statistical signal processing, machine learning and communication aspects of ITS and IoT, with an emphasis on V2X communications, localization and navigation, federated learning. She has coauthored over 130 scientific publications (journals, conferences, and patents). She is a recipient of the Marisa Bellisario Award (1999), and a co-recipient of the Best Paper Awards of the IEEE Symposium on Joint Communications and Sensing (2021), the IEEE Statistical Signal Processing Workshop (2018) and the IET Intelligent Transport Systems Journal (2014). She is an Associate Editor of the IEEE Transactions on Intelligent Transportation Systems. She also served as Associate Editor for the EURASIP Journal on Wireless Communications and Networking from 2010 to 2017, and as a Lead Guest Editor for the Special Issue on Localization in Mobile Wireless and Sensor Networks in 2011. 